\title{
PsIG, a self-produced glycosyl hydrolase, triggers biofilm disassembly by disrupting exopolysaccharide matrix
}

Shan $\mathrm{Yu}^{1,3,{ }^{*}}$, Tiantian $\mathrm{Su}^{2, *}$, Huijun $\mathrm{Wu}^{1,3}$, Shiheng $\mathrm{Liu}^{2}$, Di Wang ${ }^{1}$, Tianhu Zhao ${ }^{1,3}$, Zengjun Jin ${ }^{1,3}$, Wenbin Du ${ }^{1}$, Mei-Jun Zhu ${ }^{4}$, Song Lin Chua ${ }^{5}$, Liang Yang ${ }^{5}$, Deyu Zhu², Lichuan $\mathrm{Gu}^{2}$, Luyan Z Ma ${ }^{1}$

${ }^{1}$ State Key Laboratory of Microbial Resources, Institute of Microbiology, Chinese Academy of Sciences, Beijing 100101, China;

${ }^{2}$ State Key Laboratory of Microbial Technology, School of Life Sciences, Shandong University, Jinan, Shandong 250100, China;

${ }^{3}$ University of the Chinese Academy of Sciences, Beijing 100049, China; ${ }^{4}$ School of Food Science, Washington State University, Pullman, WA 99164-6120, USA; 'Singapore Centre on Environmental Life Sciences Engineering (SCELSE), Nanyang Technological University, Singapore 637551

Biofilms are surface-associated communities of microorganism embedded in extracellular matrix. Exopolysaccharide is a critical component in the extracellular matrix that maintains biofilm architecture and protects resident biofilm bacteria from antimicrobials and host immune attack. However, self-produced factors that target the matrix exopolysaccharides, are still poorly understood. Here, we show that PsIG, a protein involved in the synthesis of a key biofilm matrix exopolysaccharide Psl in Pseudomonas aeruginosa, prevents biofilm formation and disassembles existing biofilms within minutes at nanomolar concentrations when supplied exogenously. The crystal structure of PsiG indicates the typical features of an endoglycosidase. PslG mainly disrupts the Psl matrix to disperse bacteria from biofilms. PslG treatment markedly enhances biofilm sensitivity to antibiotics and macrophage cells, resulting in improved biofilm clearance in a mouse implant infection model. Furthermore, PslG shows biofilm inhibition and disassembly activity against a wide range of Pseudomonas species, indicating its great potential in combating biofilm-related complications.

Keywords: biofilm; glycosyl hydrolase; exopolysaccharide; PslG structure; biofilm matrix; Pseudomonas

Cell Research (2015) 25:1352-1367. doi:10.1038/cr.2015.129; published online 27 November 2015

\section{Introduction}

Structured-communities of bacteria known as biofilms are one of the most prevalent and important forms of life for bacteria in natural, industrial, and clinical settings [1, 2]. Biofilm bacteria show extreme tolerance to almost all antibiotic classes [3]. Biofilm bacteria are also protected from the host immune response, giving rise to chronic infections that are notoriously difficult to eradicate [4, 5]. One of the most important features of biofilms is extracellular polymeric substances consisting of main-

\footnotetext{
*These two authors contributed equally to this work. Correspondence: Luyan $\mathrm{Z} \mathrm{Ma}^{\mathrm{a}}$, Lichuan $\mathrm{Gu}^{\mathrm{b}}$

${ }^{a}$ E-mail: luyanma27@im.ac.cn

bE-mail: lcgu@sdu.edu.cn

Received 24 September 2015; revised 10 October 2015; accepted 10 October 2015; published online 27 November 2015
}

ly polysaccharides, proteins, and extracellular DNA (eDNA), which function as a matrix, or glue, holding biofilm cells together and protecting cells from antibiotics and shear forces in fluid environments [2, 6-8]. By forming a matrix-encased multicellular aggregate, cells can also escape engulfment by phagocytic cells within a mammalian host [9]. Hence, factors that disrupt biofilm matrix may disassemble or disperse biofilm, rendering biofilm bacteria sensitive to antibiotics and phagocytic cells. As functional and dynamic communities, biofilms are able to modify the matrix components and remodel their structures and functions as an adaptive strategy to encounter the fluctuation of various environment conditions as well as stresses. Protease and nuclease have been recently reported to be secreted by biofilm cells, which leads to disruption of protein adhesions and eDNA of biofilm matrix, resulting in remodeling of the biofilm architectures [10-12]. However, little is known about the biofilm self-generated factors that target the essential and 
universal matrix component, exopolysaccharide.

$P$. aeruginosa is an opportunistic pathogen that can cause life-threatening infections in cystic fibrosis (CF) patients and individuals with a compromised immune system $[13,14]$. This environmental bacterium can form biofilms on a variety of surfaces such as the mucus plugs of the CF lung, contaminated catheters, and contact lenses [15]. P. aeruginosa represents one of the most common agents isolated from chronic leg ulcers, often as a biofilm resistant to antimicrobial therapy [16]. Exopolysaccharides are the critical matrix component to maintain the biofilm structure of $P$. aeruginosa $[6,17]$. There are at least three exopolysaccharides, Psl, Pel, and alginate that contribute to biofilm formation in P. aeruginosa [17-20]. P. aeruginosa cells often become mucoid upon prolonged colonization of the CF lung due to the overproduction of alginate that provides an advantage for $P$. aeruginosa in the airway of CF patients [13], while non-mucoid $P$. aeruginosa strains produce little alginate and use either Pel or Psl as the primary matrix exopolysaccharide [17, 20-22]. There are 12 co-transcribed psl genes ( $p s l A B C D E F G H I J K L)$ essential for Psl production [23]. Psl, a repeating pentasaccharide containing $D$-mannose, $D$-glucose and $L$-rhamnose [23], acts as 'molecular glue' to promote bacterial cell-cell and cell-surface interactions and it can form a fiber-like web to enmesh bacterial communities $[6,7,17]$. This exopolysaccharide also confers resistance of $P$. aeruginosa biofilms to antibiotics and phagocytic cells [9, 24-26]. In P. aeruginosa PAO1 (a common lab strain), Psl is more important than Pel for biofilm microcolony formation and antibiotic resistance [24]. In addition, Psl also plays an important role in the biofilm formation of mucoid strains [27], and can function as a signal to stimulate biofilm formation [28].

Biofilms of $P$. aeruginosa develop in a five-stage multicellular cycle that is initiated by the attachment of free (planktonic) cells to a surface and finalized by seeding dispersal [2]. Our previous study implied that enzymes released from dead bacteria might degrade the exopolysaccharides matrix in the center of microcolony to free 'the seeds of biofilm' for future biofilm dispersal [6]. Here we report the discovery of one such self-generated enzyme, PslG, which is able to inhibit the biofilm formation and disrupt a pre-formed biofilm when supplied exogenously. PslG, functioning as an endoglycosidase, mainly targets Psl matrix, and shows biofilm disassembly and inhibition activity against a wide range of Pseudomonas species. Furthermore, PslG treatment sensitizes biofilm bacteria to antibiotics and macrophage cells, leading to the improved eradication of biofilms in vivo by mouse implant infection model.

\section{Results}

Overexpression of PslG inhibited biofilm formation of $P$. aeruginosa $\mathrm{PAO1}$

PslG is an essential protein for the synthesis of Psl, which is predicted to be a periplasmic protein resembling $\beta$-D-xylosidases [23, 29]. Deletion of $p s l G$ led to loss of Psl production and reduction of biofilm formation in $P$. aeruginosa PAO1 strain [23]. However, the biofilm biomass was significantly reduced in PAO1/pHERD20T-pslG, where expression of the full-length PslG was driven by $\mathrm{P}_{\mathrm{BAD}}$ promoter in plasmid pHERD20T and was induced by arabinose. Addition of $2 \%$ arabinose decreased the biofilm biomass of PAO1/pHERD20T-psl $G$ to a level similar to $\Delta p s l G$ mutant (Supplementary information, Table S1 and Figure 1A). In contrast, the biofilm biomass was increased in Psl-inducible strain WFPA801 (psl promoter was replaced by araC- $\mathrm{P}_{\mathrm{BAD}}$, Supplementary information, Table S1) due to elevated Psl production (Figure 1A), where the entire psl gene operon was induced by the $\mathrm{P}_{\mathrm{BAD}}$ promoter $[20,23]$. Given that the growth of PAO1 was not affected by PslG expression and arabinose concentration (Supplementary information, Figure S1A), these results suggested that the overexpression of PslG might decrease the production of Psl in PAO1. This was confirmed by the anti-Psl immunoblotting of Psl extracted from PAO1/pHERD20T- $p s l G$ grown with corresponding arabinose concentration (Figure 1A). These data indicated that overexpression of PslG inhibited the biofilm formation of PAO1 by reducing the production of Psl.

The purified PslG degraded Psl polymer in vitro and disrupted the Psl-fiber matrix in biofilms

How does PslG inhibit Psl production? We hypothesized that PslG as a glycosyl hydrolase might degrade the polymer of Psl when PslG was overexpressed or at a wrong location within bacterial cells or in a biofilm. To test this hypothesis, $p s l G$ lacking its signal peptide sequence was cloned from $P$. aeruginosa PAO1 and expressed in Escherichia coli. The purified $\mathrm{PslG}_{31-442}$ (containing amino acids from 31 to 442) was tested for its ability to degrade Psl in vitro. As assayed by immunoblotting, only $25 \%$ of Psl was detected $1 \mathrm{~h}$ post $\mathrm{PslG}_{31}$. ${ }_{442}$ treatment at $30{ }^{\circ} \mathrm{C}$ (Figure 1B), suggesting that the Psl polymer was degraded to smaller molecules, which cannot be detected by anti-Psl serum. $\mathrm{PslG}_{31-442}$ had the maximal Psl degradation activity at $45^{\circ} \mathrm{C}$ (Figure 1C).

Psl can form fiber-like matrix that enmeshes bacteria [6]. To investigate whether and how PslG disrupts the fibers of Psl and disperses bacteria in a biofilm, we acquired the time lapse images to track the change of Psl fibers and biofilm during PslG treatment statically at room 

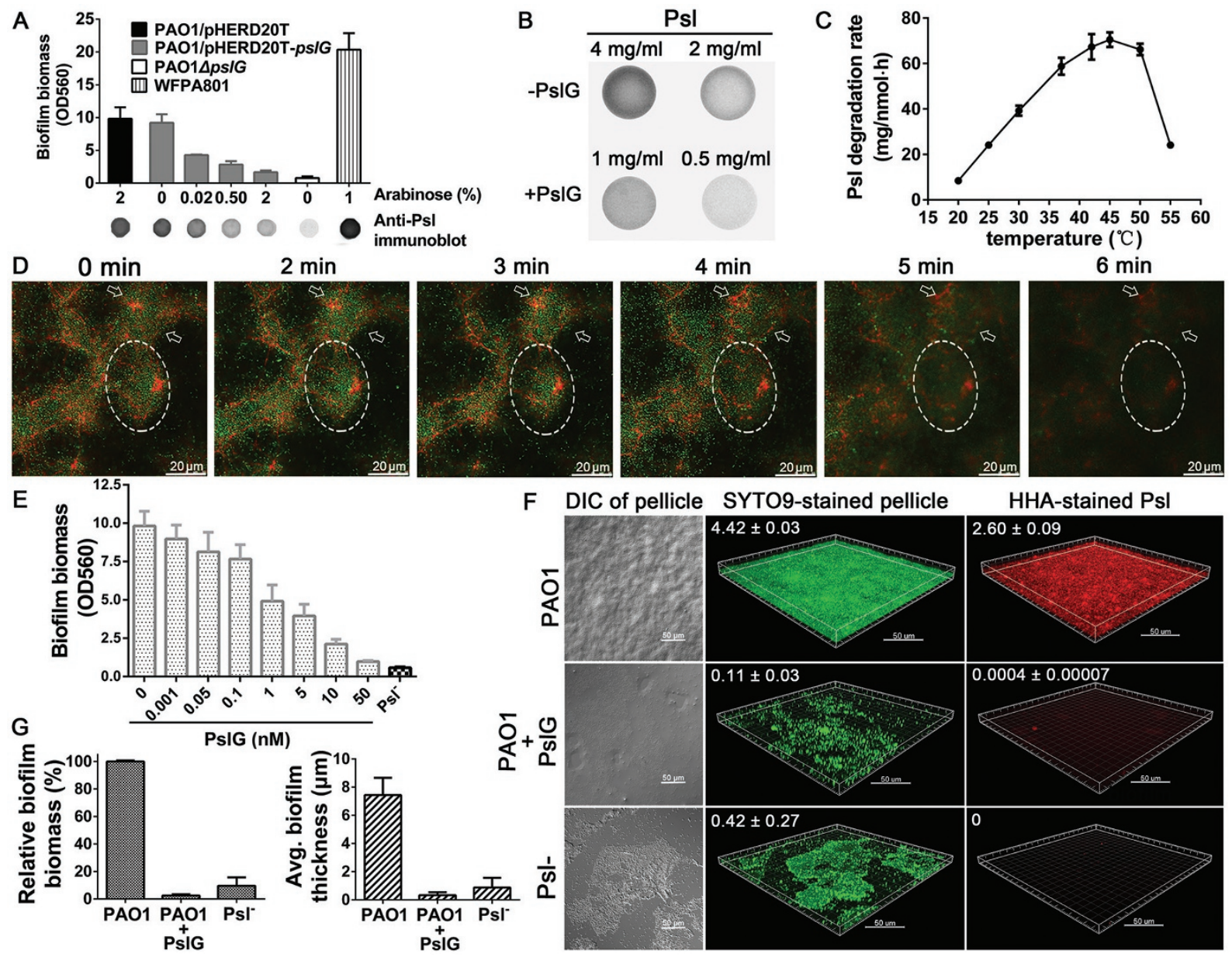

HHA-stained PsI
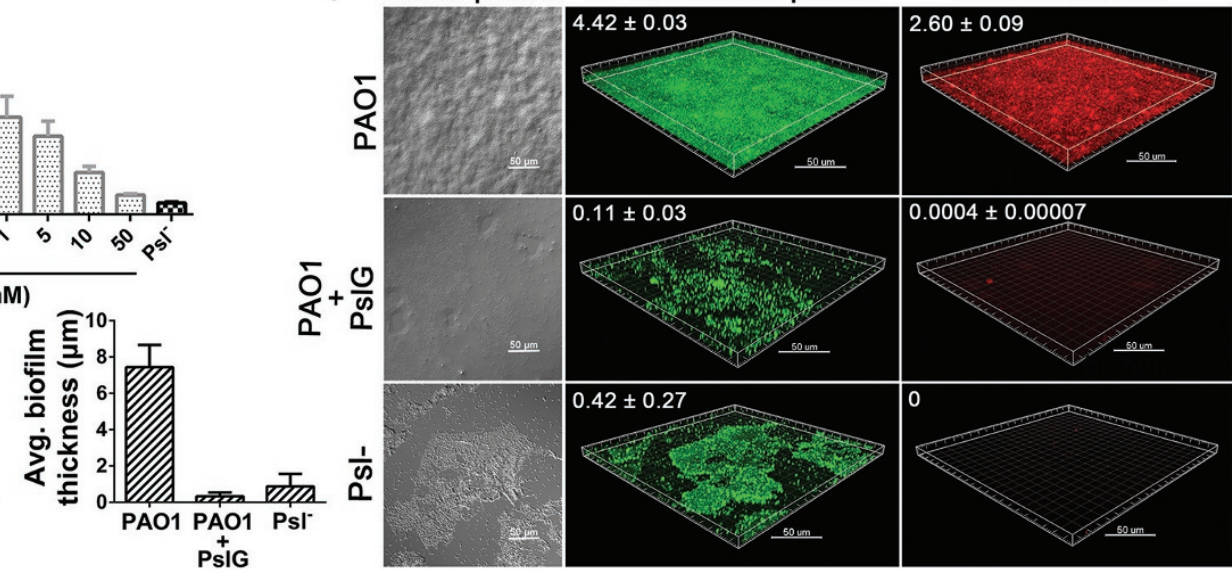

Figure 1 PsIG prevented biofilm formation by P. aeruginosa PA01. (A) The biofilm of PAO1/pHERD20T-ps/G grown with different concentrations of arabinose. Psl production was determined by anti-Psl immunoblotting as shown below each corresponding bar. (B) Purified $\mathrm{PsIG}_{31-442}$ degraded Psl in vitro. A representative dot blotting result showed the amount of Psl post 0 $\mathrm{nM}(-\mathrm{Ps} \mid \mathrm{G})$ or $50 \mathrm{nM}(+\mathrm{Ps} \mid \mathrm{G})$ PsIG treatment. The concentration of Psl was indicated above each corresponding dot. (C) The Psl degradation activity of PsIG at different temperatures. (D) Time lapse images showed that PsIG $31-442$ disrupted PsI fibers (stained in red by HHA-TRITC) in a flow-cell biofilm within 6 min (bacteria were stained in green by SYTO9, see Supplementary information, Movie S1 and Figure S2 for corresponding green and red separate images and control images); the dash lines circled a microcolony and arrows marked the edge of another microcolony. (E) $\mathrm{Ps}_{\mathrm{S}_{31-442}}$ inhibited biofilm formation in a microtiter dish assay. PAO1 was grown in media containing different concentrations of $\mathrm{Ps}_{\mathrm{IG}_{31-442}}$ and the biofilm biomass was measured by crystal violet (CV) assay $24 \mathrm{~h}$ post incubation. (F) Exogenous $\mathrm{PsIG}_{31-442}$ efficiently prevented the pellicle formation by PAO1. Images were 1-day-old pellicles grown with/without $\mathrm{PsIG}_{31-442}$ and corresponding 3-D CLSM images (bacteria in green and Psl matrix in red). (G) The quantification of biomass and average thickness of corresponding pellicles. The biomass values were normalized to that of PAO1 pellicles without $\mathrm{PsIG}_{31-442}$ (Biomass $=4.42 \pm 0.03 \mu \mathrm{m}^{3} / \mu \mathrm{m}^{2}$ ).

temperature (RT). Psl fibers (stained in red by TRITC-labeled Hippeastrum hybrid lectin from amaryllis (HHA)) began to break in 4 min after PslG was supplied (Figure 1D). Psl fibers were totally disrupted within 5 min, which was indicated by the Psl fiber-like structure turning to diffused red fluorescence (Figure 1D). Once the degradation of Psl fibers occurred (Figure 1D, 4 min), bacteria (green) in a biofilm dispersed simultaneously (Figure 1D, Supplementary information, Movie S1 and Figure S2B).
PslG prevented biofilm formation while supplied exogenously

To evaluate the biofilm-inhibiting activity, purified $\mathrm{PslG}_{31-442}$ was added to the culture media at inoculation. PAO1 biofilm biomass decreased in a PslG concentration-dependent manner (Figure $1 \mathrm{E}$ ). $\mathrm{IC}_{50}$ (the concentration that can inhibit $50 \%$ of biofilm biomass) of PslG was $\sim 1 \mathrm{nM}$ (Figure 1E). The biofilm biomass of PAO1 treated with $50 \mathrm{nM} \mathrm{PslG} 31-442$ was similar to that of the 
Psl-negative strain WFPA800 ( $p s l$ promoter deletion strain, Supplementary information, Table S1 and Figure $1 \mathrm{E})$, indicating that PslG at $50 \mathrm{nM}$ can completely prevent Psl-dependent biofilm formation.

P. aeruginosa can form floating biofilms known as pellicles at the air-liquid interface of standing cultures. We further tested the inhibitory effect of $\mathrm{Ps}_{\mathrm{s}} \mathrm{G}_{31-442}$ against pellicle formation of PAO1. The biomass of PAO1 pellicle grown in the presence of $50 \mathrm{nM}$ PslG was even lower than Psl-negative strain, indicating that Psl-dependent pellicle formation was totally prevented (Figure $1 \mathrm{~F}$ and $1 \mathrm{G}$ ). Consistently, fluorescence-labeled lectin HHA staining showed that the pellicles with PslG treatment had little Psl matrix (Figure 1F, red) and $99.9 \%$ of Psl in pellicles was degraded. The biofilm inhibitory effect of $\mathrm{PslG}_{31-442}$ was not due to change of bacterial growth because planktonic PAO1 showed similar growth rate regardless of PslG concentration (Supplementary information, Figure S1B). In addition, PslG was also unlikely to inhibit the growth of biofilm bacteria or to change their physiology because PslG showed little impact on the GFP expression in the biofilm cells of PAO1/pCdrA::gfp ${ }^{\text {s }}$ during 3 $\mathrm{h}$ of monitoring (Supplementary information, Table S1 and Figure S1D). Plasmid pCdrA::gfp is also a reporter for cyclic-di-GMP level in $P$. aeruginosa, which is an important signal molecule involved in the regulation of biofilm formation [30]. Thus this result indicated that the level of cyclic-di-GMP in the biofilm of PAO1/pCdrA::gfp was not affected by $3-\mathrm{h}$ PslG treatment. Altogether, the data suggested that the PslG-mediated biofilm inhibition was not due to the change of intracellular cyclic-di-GMP level or the inhibition of bacterial growth.

\section{Exogenous PslG disassembled existing biofilms}

The biofilm of $P$. aeruginosa PAO1 primarily used Psl to maintain its community structure $[17,31]$. We therefore tested the biofilm disassembly activity of PslG on existing biofilms of PAO1. Addition of $1 \mathrm{nM} \mathrm{PslG}_{31-442}$ reduced half of biomass within $30 \mathrm{~min}$ at $30^{\circ} \mathrm{C}$ (Figure 2A and $2 \mathrm{~B}$ ). At this PslG concentration, the biofilm biomass decreased continually for $8 \mathrm{~h}$ (Figure 2B, grey triangle). Furthermore, the biofilm disassembly activity of PslG increased with concentration (Figure $2 \mathrm{~A}$ ). $\mathrm{Ps}_{31-442}$ at 50 $\mathrm{nM}$ dispersed $50 \%$ of biofilm in $10 \mathrm{~min}$ and $82 \%$ of bio-
A

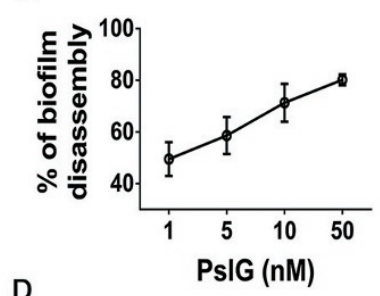

D

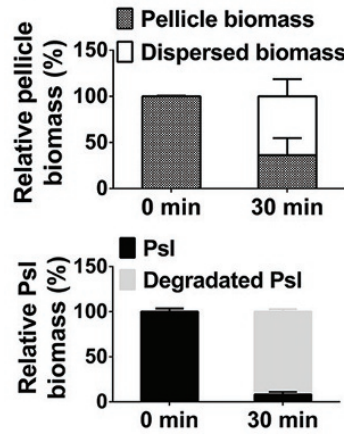

B
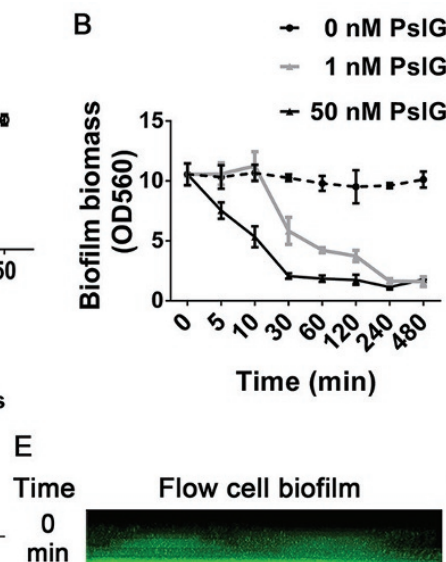

30

180

min

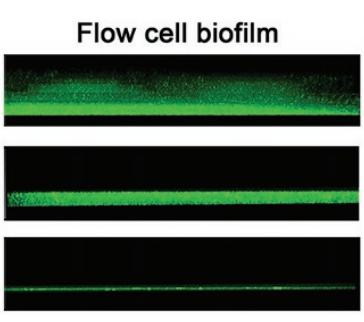

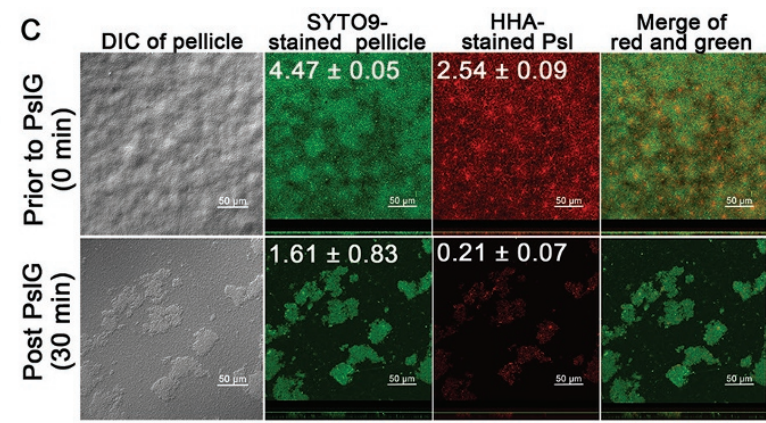

$F$

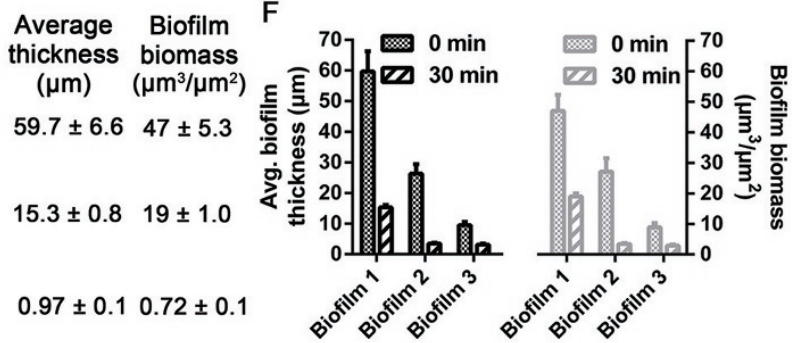

Figure 2 PsIG triggered biofilm disassembly. (A) PsIG P1-442 disassembled pre-formed PAO1 biofilms in a dose-dependent manner. Disassembly rate was calculated by ratio of biofilm biomass post $30 \mathrm{~min}$ of PsIG treatment to that of untreated control. (B) Time course of $\mathrm{PsIG}_{31-442}$ effects on biofilm disassembly. The biofilm biomass was measured by CV assay. (C) Representative optical section images showing the 1-day-old PAO1 pellicles and their corresponding Psl matrix prior to (0 min) and post 30 min of PsIG treatment. The horizontal sectioned images (square) and vertical sectioned images were shown. The biomass and scale bar were indicated on the corresponding image. (D) Quantification of pellicle biomass and PsI matrix. The values were normalized to the biomass of pellicles before PsIG treatment (0 min). (E) PsIG disassembled biofilm microcolonies formed in a flow cell chamber. Representative vertical sectioned images showed the change of microcolonies during $3 \mathrm{~h}$ of PsIG treatment. Corresponding biomass and thickness were indicated next to the images. (F) Comparison of biomass and thickness prior to $(0 \mathrm{~min}$ ) and post $30 \mathrm{~min}$ of PsIG treatment for three flow-cell biofilms with varied thickness. 
film within $30 \mathrm{~min}$ (Figure 2B, black triangle). Treatment time beyond $30 \mathrm{~min}$, however, did not further reduce the biofilm biomass, yet the biomass remained at a low level during $8 \mathrm{~h}$ of incubation (Figure 2B, black triangle). This suggested that Psl-dependent biofilms were abolished within $30 \mathrm{~min}$ by $50 \mathrm{nM}$ PslG. Similarly, PslG (at 50 $\mathrm{nM}$ ) reduced $55 \%-82 \%$ biomass of 1-day-old pellicles in 30 min (Figure $2 \mathrm{C}$ and 2D). There was only $8 \%$ Psl remaining with biofilm post treatment, indicating that $92 \%$ of matrix Psl was disrupted (Figure 2D). PslG showed the maximal disassembly activity at $45{ }^{\circ} \mathrm{C}$ (Supplementary information, Figure S3), which was consistent with its Psl degradation activity in vitro (Figure 1C).

DNase I was reported to disrupt a 'young' biofilm of $P$. aeruginosa by targeting matrix eDNA, yet it could not disrupt biofilms of PAO1 once microcolonies formed in a flow cell chamber [32]. To know whether PslG can disrupt pre-formed microcolonies, we grew the biofilm of PAO1 in a flow cell chamber with continuous medium flow for $36 \mathrm{~h}$, and then treated with $50 \mathrm{nM} \mathrm{PslG} \mathrm{Bl}_{31-442}$ for up to 180 min statically. PslG can disassemble microcolonies and biofilms with varied thickness and $55 \%-80 \%$ of biomass was dispersed by PslG in 30 min (Figure 2E and 2F). Regardless of the thickness of biofilms before treatment, some biomass remained on the glass surface after 30 min of PslG treatment (Figure 2F). It required another $3 \mathrm{~h}$ of PslG treatment to remove the remaining biofilm biomass (Figure 2E).

The crystal structure of PslG indicated typical features of an endoglycosidase

We crystallized PslG and determined its crystal structure at a resolution of $2.0 \AA$ (Table 1). The refined structure showed that four PslG molecules were loosely packed in one asymmetric unit with less than $1100 \AA^{2}$ contacting surface between each monomer, which covered only $6.5 \%$ surface area of the PslG monomer. Thus they unlikely form a functional tetramer. This was supported by the results of dynamic light scattering (DLS), which showed that $\sim 97 \%$ of PslG molecules existed as

Table 1 Crystal structure data collection and refinement statistics

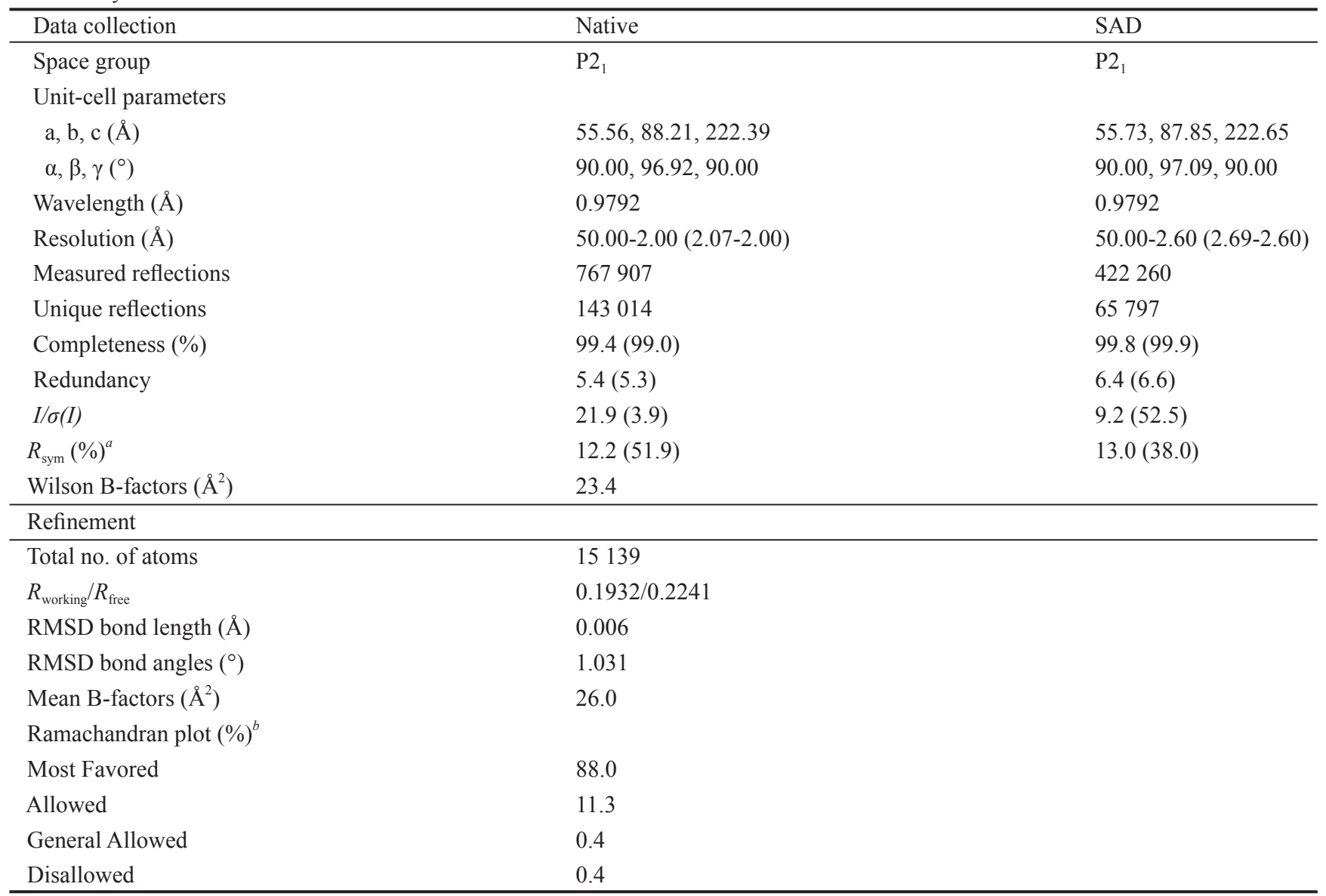

${ }^{a} R_{\text {sym }}=\Sigma_{h k l} \Sigma_{i}\left|I_{i}(h k l)-<I(h k l)>\right| / \Sigma_{h k l} \Sigma_{i} I_{i}(h k l)$, where $<I(h k l)>$ is the mean intensity for multiply recorded reflections.

${ }^{b}$ As defined in PROCHECK. 
monomer form in solution (Supplementary information, Figure S8C). Each PslG monomer contains 412 residues (31-442), which folds into two domains, a large and a small domain (Figure 3A). PslG was predicted to be a $\beta$-D-xylosidase from the CAZy Glycosyl Hydrolase Family 39 [29], which usually exists as tetramers in solution and contains three distinct domains. However, PslG lacked the small $\alpha$-helical domain and the catalytic $\beta$-hairpin present in the hydrolases in Family 39. An online search against Protein Data Bank (PDB) using DALI server [33] showed that the structure of a Xylanase from Erwinia chrysanthemi (ECXyna, PDB: 1NOF; Z-score: 26.7), belonging to Glycoside Hydrolase Family 5, was most similar to PslG although it only had $13 \%$ sequence identity with PslG [34]. We designated the large domain of PslG as the catalytic domain and the small domain as the carbohydrate-binding domain (CBM; Figure 3A) by analogy to ECXyna (Supplementary information, Figure S6A).

The catalytic domain of PslG includes residues 47358 and adopts the common $(\beta / \alpha)_{8}$-barrel fold (Figure
$3 \mathrm{~A}$ and $3 \mathrm{~B}$ ), which is conserved in polysaccharide hydrolase families. Surface analysis indicated an extensive substrate-binding groove which traversed the whole catalytic domain and was large enough to accommodate a polysaccharide chain (Figure 3D and 3E), as featured by a typical endoglycosidase [35]. Sequence alignment and structure comparison with homologous proteins identified through DALI suggested that Glu165 and Glu276 of PslG were the key catalytic residues (Figure 3B). Glu165 is located on one side of the cleft with the carboxyl group of its side chain pointing toward the opposite side (Figure 3B). Such a positioning may allow this residue to serve as acid or base during catalytic process [34]. In contrast, Glu276 is positioned on the bottom of the cleft with the side chain carboxyl group pointing upward (Figure 3B), suggesting that this residue may function as the nucleophile during catalytic reaction [34]. The two residues are separated by a distance of $4.1 \AA$, which is close to the average distance $(\sim 5.5 \AA)$ between the two catalytic residues in retaining enzymes (in contrast to $\sim 10 \AA$ in inverting enzymes) [36,37]. These results suggest
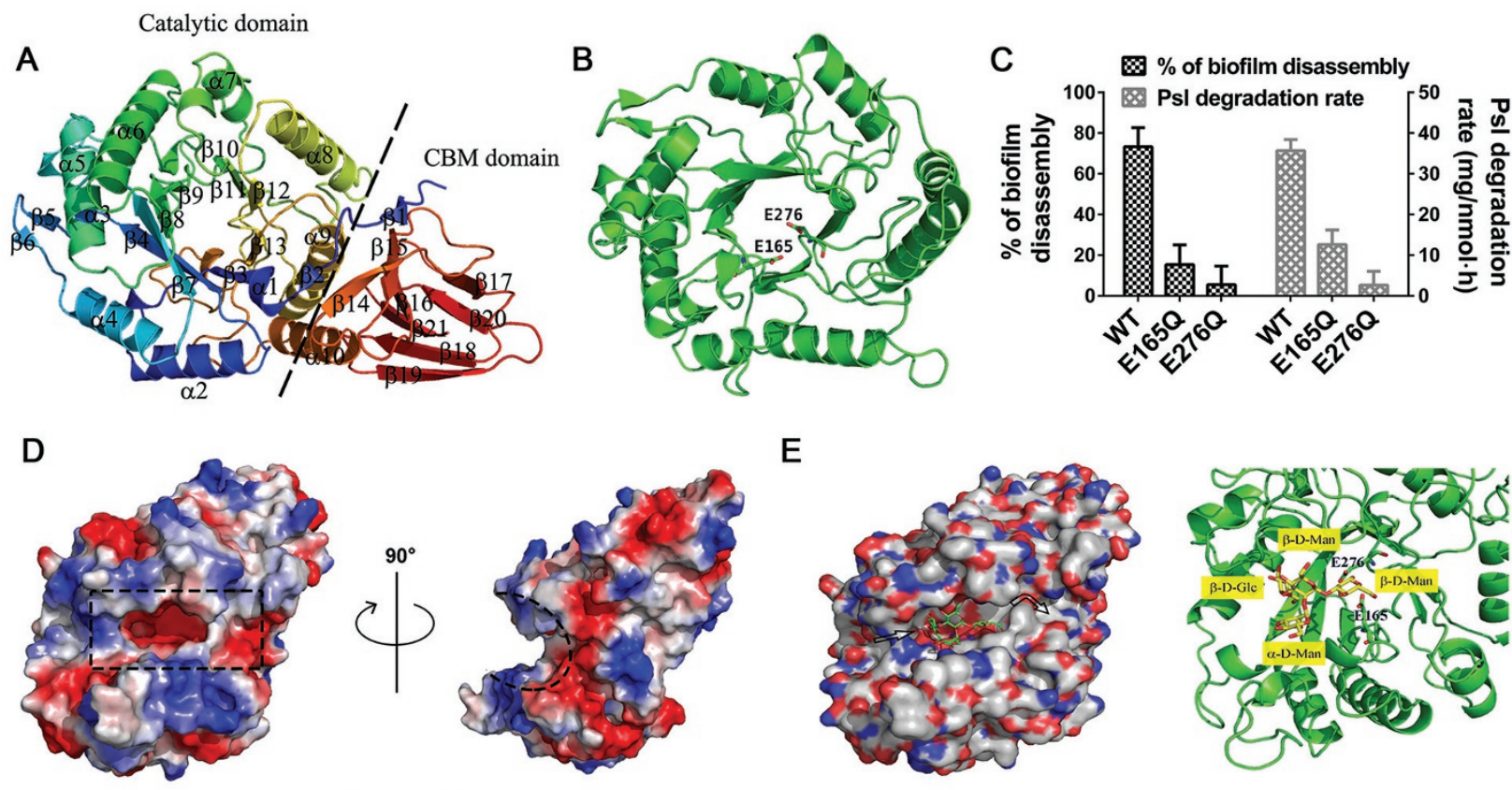

E
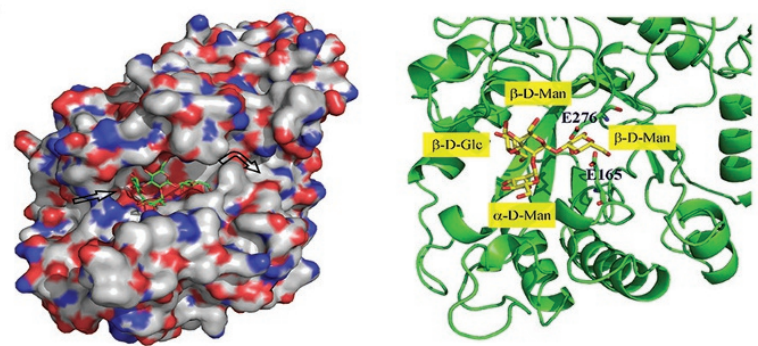

$-61.870 \quad 51.870$

Figure 3 Crystal structure of PsIG. (A) The structure of PsIG was represented in cartoon and colored in rainbow mode. The dashed line separated the catalytic domain and carbohydrate-binding domain (CBM). (B) The key catalytic residues (E165 and E276) in catalytic domain of PsIG were shown in stick. (C) Mutation on either E165 or E276 significantly reduced PsI degradation and biofilm disassembly activity of PsIG at $30{ }^{\circ} \mathrm{C}$. (D) Electrostatic potential of the PsIG catalytic domain observed from different perspectives. The regions with negative or positive charge are colored in red or blue, respectively. The catalytic site and cleft was marked by dash line. (E) The Autodock analysis showed how PsIG bound with Psl. A possible

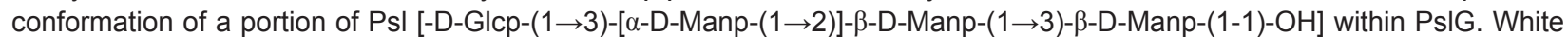
arrows indicated the possible binding groove for Psl. 
that PslG is likely a retaining enzyme. The importance of these two residues was confirmed by a mutagenesis study, which showed that E165Q or/and E276Q mutation led to a significant decrease in the catalytic activity of PslG with regard to Psl degradation and biofilm disassembly (Figure 3C and Supplementary information, Figure S4B). Consistently, the corresponding PslG mutants showed a reduced or no activity of inhibiting the biofilm formation and decreasing Psl production when overexpressed in PAO1 (Supplementary information, Figure S4C). Through molecule docking, we proposed a model that represented the binding of a portion of Psl repeat unit containing 3 mannoses and 1 glucose to the catalytic site (Figure 3E). This model suggests that PslG might cleave the bond between $\beta$-D-Man and $\alpha-L$-Rha (Supplementary information, Figure S5). There are 27 other residues involved in the formation of the two catalytic cleft walls (Supplementary information, Figure S6C) [34]. Of these residues, 13 hydrophobic residues may serve as the scaffold to dock the pyranose rings of the polysaccharide chain and 14 other residues offer hydrogen bonds to stabilize the polysaccharide chain and ensure substrate specificity of PslG (Supplementary information, Figure S6C). We constructed four point mutations among these residues: D79R, D133Y, F208A and F208W. All the mutants showed a reduced activity of Psl degradation and biofilm disassembly compared to the wild-type PslG (Supplementary information, Figure S6D), suggesting their involvement in the substrate binding. Electrostatic potential analysis showed that the catalytic cleft of PslG was negatively charged, and this region was much deeper and more widely opened than that in ECXynA (Figure 3D and Supplementary information, Figure S6). Although PslG has an overall structure highly similar to ECXynA, most of the residues lining the walls of the substrate-binding cleft are not conserved in these two proteins. In contrast, sequence alignment showed that most residues for the substrate-binding cleft were conserved among the PslG homologues from Pseudomonas species (Supplementary information, Figure S7). This suggested that PslG might also be used to treat biofilm infection caused by Pseudomonas species other than $P$. aeruginosa.

The CBM of PslG consists of nine $\beta$-strands, of which the first one is composed of the first 12 residues (31-42) of the mature protein. The other eight $\beta$-strands contain residues 361-442. All these residues of the CBM domain fold into a tetrahedral shape (Figure 3A). The CBM domain is covalently connected to the catalytic domain through two very short linker peptides (residues 43-46 and 359-360) and an extensive hydrophobic interface formed between these two domains. The potential of CBM domain to bind carbohydrates was supported by the behavior of PslG on gel filtration column. When loaded onto a superdex 200 column, PslG (47 kDa) gave an abnormal large elution volume of $17 \mathrm{ml}$ that generally corresponded to a salt peak (Supplementary information, Figure S8A). Increasing of the salt concentration in elution buffer did not reduce the elution volume of PslG (Supplementary information, Figure S8B). In contrast, xylanase PtXyI43 (38 kDa), a $\beta$-1,4-xylosidase from Paecilomyces $s p$. J18 with only a catalytic domain had a normal elution volume consistent with its molecular size (Supplementary information, Figure S8A). Elution delay suggests that PslG has some binding affinity to dextran, the major component of superdex 200 column.

PslG inhibited biofilm formation and disrupted existing biofilms of a wide range of Pseudomonas strains

To investigate the specificity of PslG, we examined the inhibition and disruption activity of PslG against biofilms of many strains. We first investigated several PAO1-derived mutant strains, such as Psl- or Pel-negative strains, Psl-inducible strain WFPA801 (PAO1, $\mathrm{P}_{\mathrm{BAD}^{-}}$ $p s l$ ), mucoid strain PDO300 (alginate overproducer), and a rugose small-colony variant (RSCV) MJK8 that displays increased production of the Pel and Psl [38]. PslG significantly inhibited the biofilm formation of all PAO1-derived strains, which included Psl-negative strains and MJK8-derived $\Delta p s l \Delta p e l$ strain (Figure 4A). The prevention of biofilm formation by PslG was not a result of growth defect because PslG did not affect the growth rate of Psl-negative strain, Psl overproducer, Pel overproducer, and alginate overproducer (Supplementary information, Figure S1C). PslG also disrupted the established biofilms of most tested PAO1-derived strains (except for PDO300 $\Delta p s l$ and MJK8 $\Delta p s l \Delta p e l$, data not shown) with a disassembly rate ranging from $20 \%$ to $82 \%$ (Figure 4B). These data suggested that PslG had some but limited degradation activity on polysaccharides other than Psl.

We next tested the efficacy of PslG against 26 environmental and clinical $P$. aeruginosa isolates that produced different amount of Psl (Table 2). PslG disassembled preformed biofilms of most tested strains and prevented their biofilm formation to some degree, except for the strain PA14 (a strain had Pel-dominant matrix) and seven $\mathrm{CF}$ isolates that produced little Psl (Table 2). PslG homologues were highly conserved in Pseudomonas species (Supplementary information, Figure S7), thus we examined the effect of PslG on the biofilm of P. syringae pv. phaseolicola and P. stutzeri A1501. PslG showed biofilm inhibition and disassembly activity against the plant pathogen P. syringae pv. phaseolicola (Figure 4C). PslG prevented the pellicle formation of $P$. stutzeri A1501 

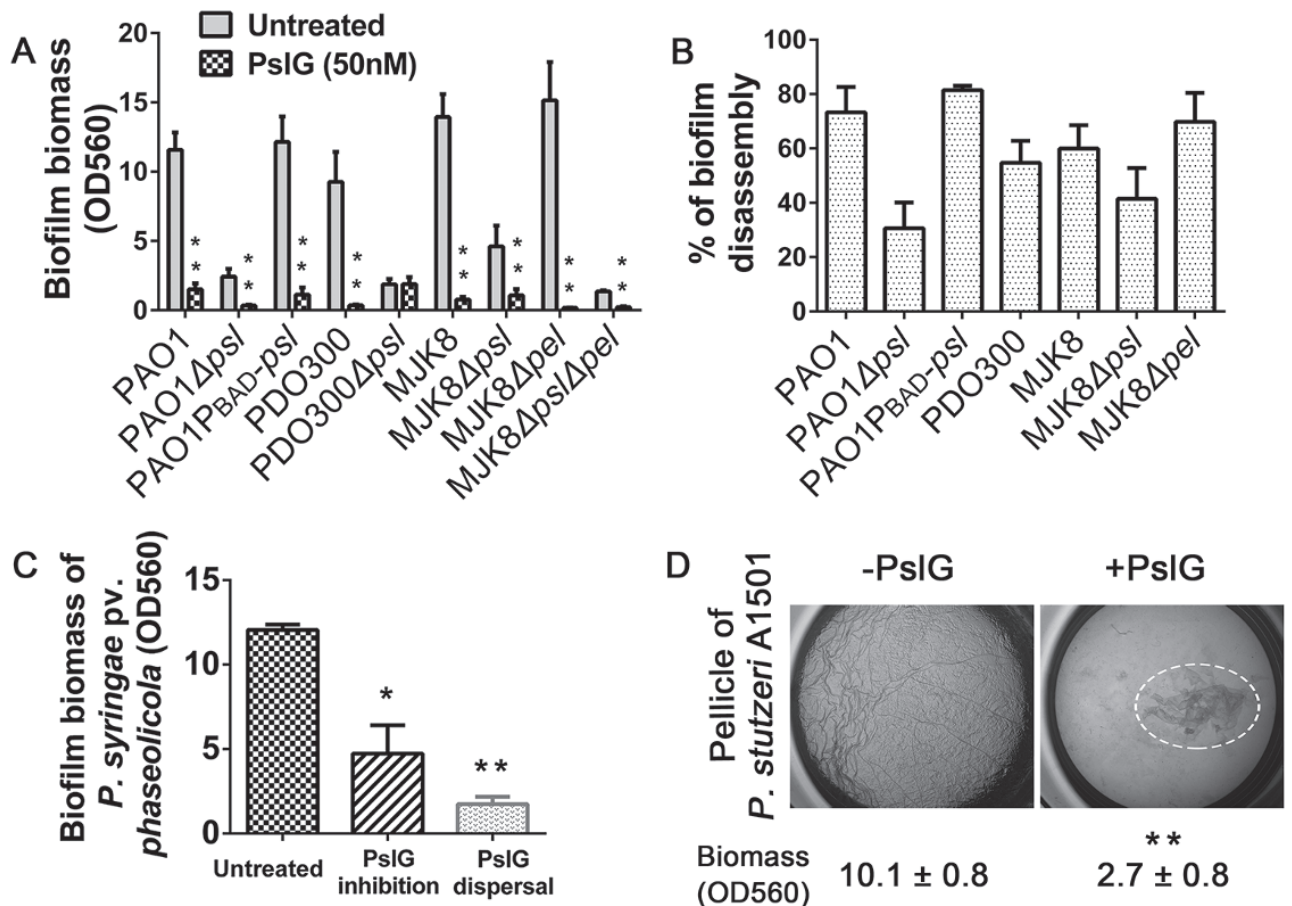

Figure 4 Effects of PSIG on the biofilms formed by different Pseudomonas strains. (A, B) Biofilm inhibition and disassembly activity of PsIG against $P$. aeruginosa PAO1-derived mutant strains. (C) The effect of PsIG on the biofilms formed by $P$. syringae pv. phaseolicola. (D) The inhibitory effect of PsIG on the pellicles of $P$. stutzeri A1501; the remaining pellicle post PsIG treatment was marked by dash line. $T$-test was performed for testing differences between groups. ${ }^{*} P<0.01 ;{ }^{* *} P<0.001$.

(Figure 4D), yet could not disrupt an existing biofilm of this strain. PslG had little effect on the biofilms of Staphylococcus aureus, Candida albicans, E. coli, and Salmonella enteric (Supplementary information, Figure S9).

PslG treatment sensitized biofilm bacteria to antibiotics and macrophage cells

To investigate whether PslG can improve the sensitivity of biofilm bacteria to antibiotics, we tested the MIC (minimum inhibition concentration) of two selected antibiotics commonly used to treat $P$. aeruginosa infections, Tobramycin and Ciprofloxacin against PslG-dispersed bacteria from 1-day-old biofilms. PslG-dispersed bacteria had a slightly lower MIC than planktonic bacteria (Figure $5 \mathrm{~A}$ ). To know whether antibiotics can eradicate the remaining surface-attached biofilm after $30 \mathrm{~min}$ of PslG disruption, we applied the Calgary biofilm device [39] to examine the MBEC (minimum biofilm eradication concentration). The MBEC of biofilms post PslG treatment was 4-fold (Cip) or 8-fold (Tob) lower than those of non-treatment controls (Figure 5B). The MIC of antibiotics against the planktonic growth from peg-attached biofilms was also tested. MIC of bacteria from PslG-treated biofilm was 2-fold lower than that of untreated biofilms (Figure 5B). To investigate whether there is a synergistic effect of PslG and antibiotics, and the efficacy of PslG at a limited delivery condition, we used the microfluidic devices (Figure 5C), in which the total PslG used during 5-h continuous delivery was half of the other systems (such as microtiter and flow cell chambers). The biofilm biomass (calculated from fluorescent intensity of GFPtagged PAO1) in PslG-treated group decreased by 5 -fold in $5 \mathrm{~h}$ (Figure $5 \mathrm{C}$ ), while the biofilm biomass in untreated control doubled in $5 \mathrm{~h}$ (Figure 5C). Addition of antibiotic (either Tob or Cip) to PslG-treated group did not further reduce the biomass (Figure 5C), suggesting that the tested antibiotics did not affect the biofilm disassembly activity of PslG, yet there appeared no synergistic effect in this system. Given that PslG did not have any effect on the anti-Pseudomonas activity of Tob or Cip as indicated by an agar diffusion assay and MIC tests (Supplementary information, Figure S10), we concluded that PslG treatment can be coupled with antibiotic therapy to combat the biofilm-related infections.

To test whether PslG has any toxic effects to mammal cells, we used the human colonic epithelial cell line Caco2 or HT-29 to test the cytotoxicity of PslG. Tetra- 
Table 2 The efficacy of PslG against the biofilms formed by clinical and environmental $P$. aeruginosa isolates

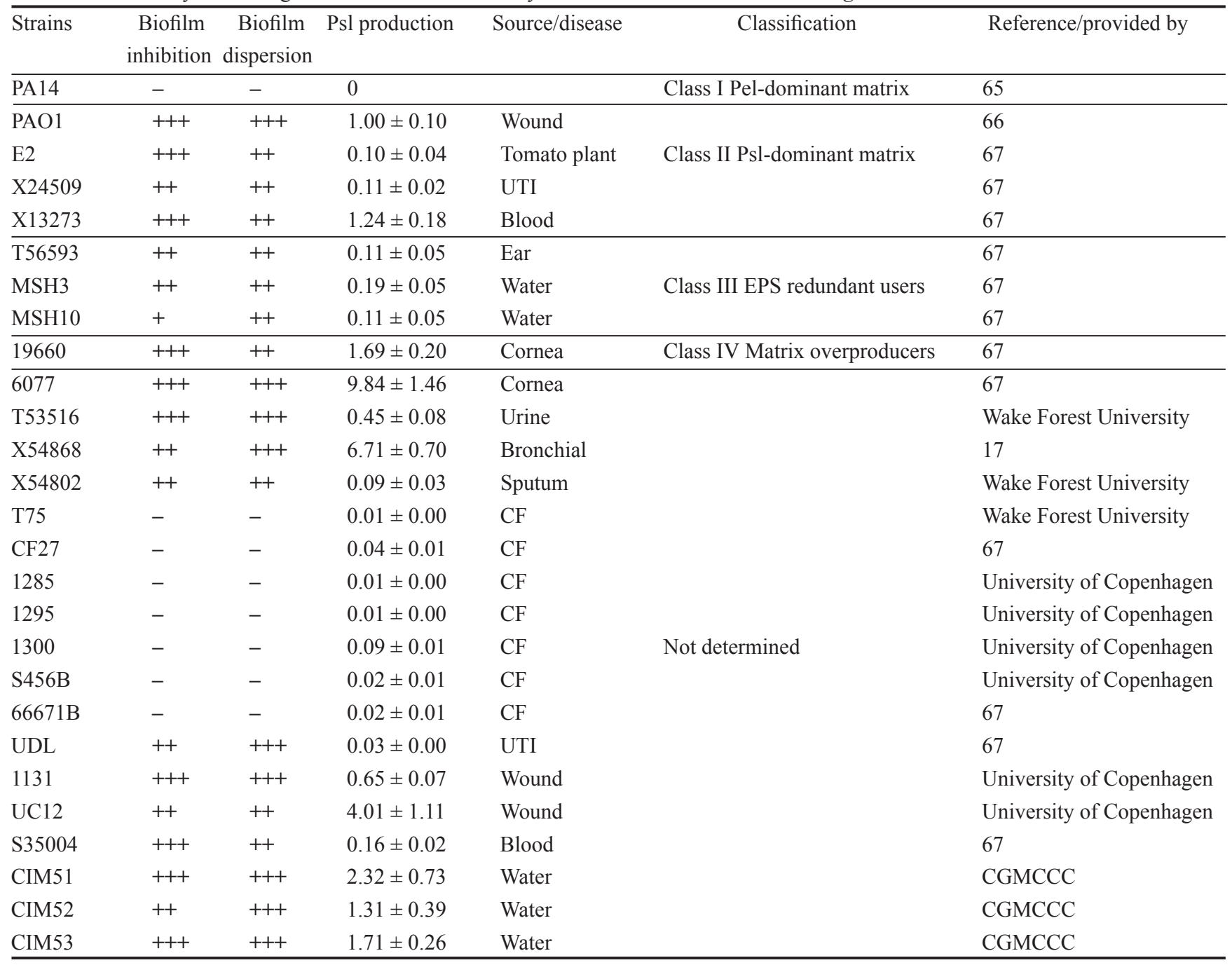

"+++", the reduction of biofilm biomass by PslG is above 70\%; "++", the reduction of biofilm biomass is $30 \%-70 \%$; "+", the reduction of biofilm biomass is less than $30 \%$; “-”, no significant differences between biofilms with and without treatment. The values of Psl have been normalized to the result of PAO1 (32-45 $\mu \mathrm{g} / \mathrm{ml}$ crude Psl). CGMCCC: China General Microbiological Culture Collection Center.

zolium MTT cytotoxicity assay showed that PslG had minimal effect on the viability of both colonic epithelial cells (Supplementary information, Figure S11A), neither did PslG show any cytotoxicity to macrophages cells (RAW264.7; Supplementary information, Figure S11B). As Psl was reported to confer resistance of $P$. aeruginosa biofilms to phagocytic cells [25], we further tested whether PslG could improve the sensitivity of biofilm bacteria to macrophages. Indeed, PslG treatment rendered biofilm bacteria more susceptible to macrophages, indicated by the least bacteria detected post the treatment of PslG coupled with macrophages (Figure 5D). Taken together, our data indicated that PslG was not toxic to host cells and PslG treatment sensitized biofilm bacteria to antibiotics and phagocytes, suggesting the potential application of PslG in treating biofilm-related infections.

\section{PslG improved the eradication of biofilms in vivo}

We used a murine model for implant-associated infections to further determine whether PslG could assist in the eradication of biofilms in vivo. Implants coated with $P$. aeruginosa biofilms were inserted into the mouse peritoneum and treated locally with the following treatments: $50 \mathrm{nM}$ PslG; $50 \mathrm{mg} / \mathrm{kg}$ tobramycin; and $50 \mathrm{nM}$ PslG plus $50 \mathrm{mg} / \mathrm{kg}$ tobramycin. After $24 \mathrm{~h}$ of incubation in the mice, the implants were removed for homogenization and enumeration of bacteria. Either PslG or tobramycin treatment was able to reduce the bacterial load on the implant as compared to the control. Furthermore, the 


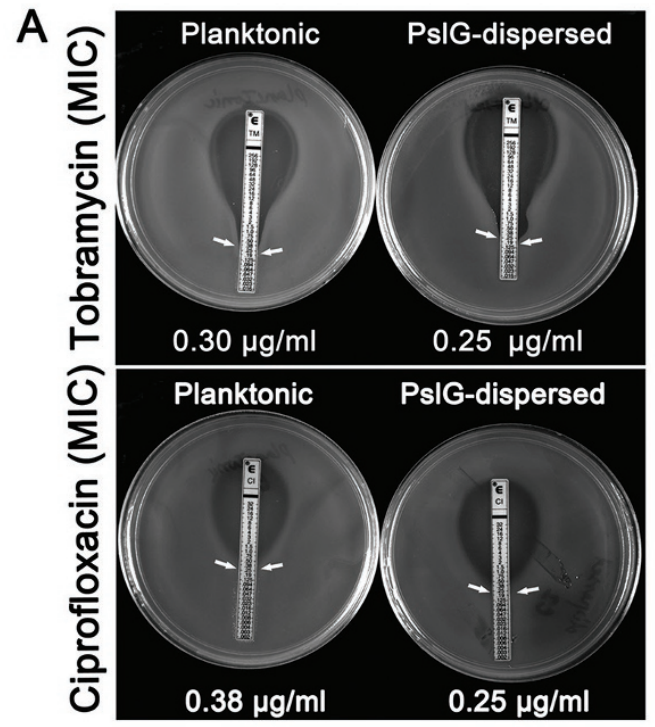

MIC
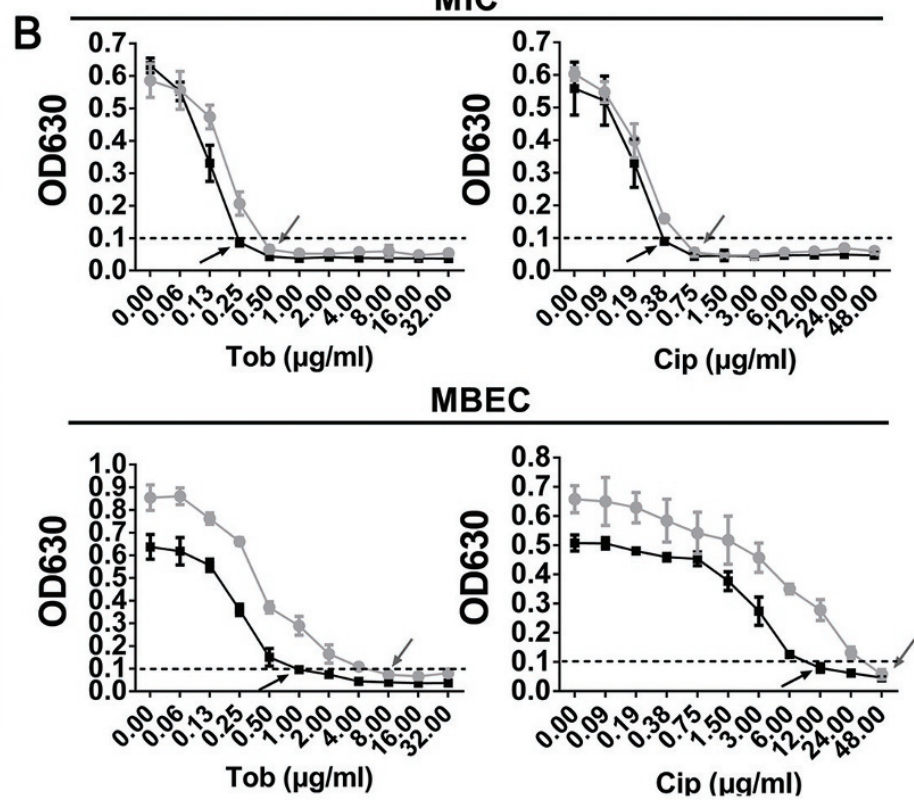

C
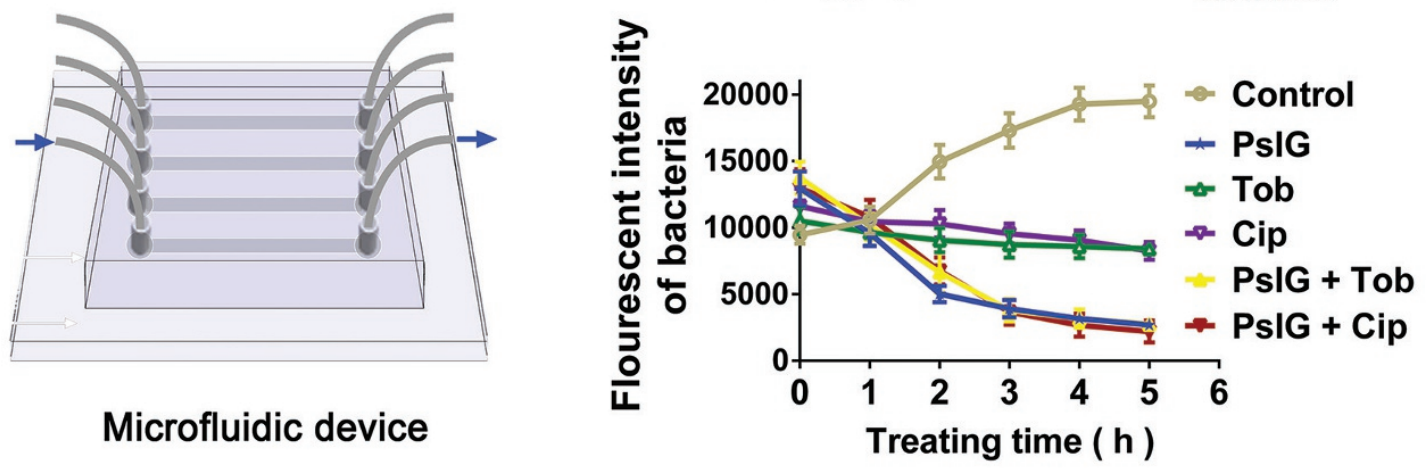

D

\section{Microfluidic device}

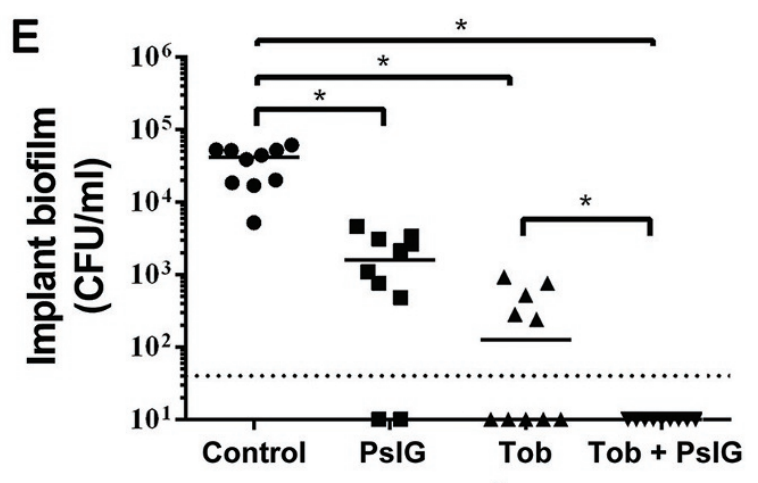

Figure 5 PsIG treatment improved biofilm sensitivity to antibiotics and phagocytic cells. (A) E test strip results. PsIG-dispersed bacteria from a biofilm were more sensitive to tobramycin (Tob) and ciprofloxacin (Cip) than planktonic cells. Arrows indicated the corresponding MIC values on the strip. (B) MBEC and MIC of biofilms treated with ( $\bullet$ ) and without (•) PsIG. Arrows indicated the corresponding values of MBEC and MIC. (C) Tob and Cip did not affect PsIG biofilm disassembly activity. GFP-tagged biofilms were monitored in microfluidic devices (left) with continuous media flow supplied with PsIG or PsIG combining with either Tob or Cip. Blue arrows indicated the flow direction. (D) PsIG enhanced biofilm sensitivity to macrophage cells. PAO1 biofilms were cultivated on top of glass cover slides for $24 \mathrm{~h}$ and treated with PsIG and macrophage cells. Biofilms were then scraped off the glass slides and re-suspended in $1 \mathrm{ml} 0.9 \% \mathrm{NaCl}$ for colony forming unit (CFU) determination. Means \pm SD in CFU/ml from triplicate experiments are shown. (E) PsIG improved host clearance of implant biofilms and showed combinatory effect with Tob in eradication of implant biofilms in vivo. The median value was indicated. $T$-test of unpaired unequal variance was performed for testing differences between groups. ${ }^{*} P<0.03,{ }^{* *} P<0.01$. 
synergistic treatment of PslG and tobramycin led to more significant reduction in the bacterial load on the implant compared to the single treatment groups (Figure 5E). This showed that the addition of PslG could be possibly used clinically to treat biofilm infections.

\section{Discussion}

Exopolysaccharides are a common component of the extracellular matrix in biofilms formed by many Gram-positive, Gram-negative bacteria and fungus $C$. albicans [6, 40-42]. In this study, we report that PslG, a glycoside hydrolase, prevents the biofilm formation of Pseudomonas species and efficiently disrupts their existing biofilms at nanomolar concentrations. Glycoside hydrolases are common enzymes in nature with a variety of roles. However, few enzymes were reported to disassemble an existing biofilm at nanomolar concentrations like PslG. Cellulase has been reported to have some biofilm inhibition activity by partially degrading Psl and Pel in $P$. aeruginosa $[6,22]$. A secreted $N$-acetylglucosaminidase, $\mathrm{DspB}$, has been shown to cause detachment and dispersion of Actinobacillus actinomycetemcomitans biofilm cells $[43,44]$. However, cellulase and DspB function at millimolar or micromolar concentrations. $D$-amino acids have been reported to disassemble the biofilms of Bacillus subtilis by targeting matrix proteins [40], yet a later report showed that this is due to an indirect effect of inhibition of protein synthesis and bacterial growth [45]. Thus far, to the best of our knowledge, PslG is the most efficient factor for biofilm disassembly and inhibition without affecting bacterial growth.

One of the most important causes of starvation-induced tolerance in vivo is biofilm growth $[4,5,46]$. Starvation in biofilms is attributed to nutrient consumption by cells located on the periphery of biofilm and to reduced diffusion of substrates through the biofilm. Enzymes or factors that can disrupt biofilm matrix not only disassemble bacterial community structure, but also may allow substrates or antibiotics to diffuse into biofilm, leading to improvement of biofilm clearance. Consistent with this concept, we have shown that PslG treatment remarkably sensitizes biofilm bacteria to antibiotics and macrophage cells. Several lines of evidence indicate the great potential of PslG in combating biofilm. First, PslG is not toxic to human epithelial cells and immune cells; second, PslG can be used with antibiotics to eradicate biofilms; third, PslG shows biofilm inhibition and disassembly activity against a wide range of Pseudomonas species; fourth, PslG-dispersed bacteria from PAO1 biolfilms are not more virulent than their planktonic counterparts as revealed by the $C$. elegans liquid killing assay (Supplementary information, Figure S11C). Finally and most strikingly, PslG does not inhibit the growth of $P$. aeruginosa regardless of the type of exopolysaccharides or the amount of exopolysaccharides that were produced (Supplementary information, Figure S1). In addition, PslG shows little impact on the physiology of bacteria within biofilms regarding the gene expression and the level of an important second messenger cyclic-di-GMP.

PslG exhibits biofilm inhibition activity on several PAO1-derived Psl-negative strains (Figure 4A) and several clinical or environmental strains producing little Psl (Table 2). Consistently, pellicles grown with PslG have even lower biomass than Psl-negative strain (Figure 1F). These data suggest that PslG might hydrolyze exopolysaccharides other than Psl. However, PslG is not likely to hydrolyze alginate because the inhibition on mucoid biofilms is mostly due to the degradation of Psl according to the result that PslG does not inhibit the biofilm formation of Psl-negative mucoid strain PDO300 $\Delta$ psl. The CBM domain of typical endoglycosidases is usually involved in the substrate binding, such as endocellulases, which have a long shallow groove on the surface of CBM domain for polysaccharide substrate binding besides the deep groove in catalytic domain for the polysaccharide substrate to fit into [47]. However, the CBM domain of PslG is significantly different from the classic CBM of endoglycosidases, which lacks the aromatic residue cluster lined groove for substrate binding. Further investigation will be helpful to clarify whether this typical feature leads to the affinity of PslG with some other kinds of carbohydrates except Psl or is involved in Psl synthesis.

PslG is required for the production of Psl [23], yet how exactly PslG is involved in the biosynthesis of Psl is still unclear. Franklin et al. [29] has predicted that PslG might be responsible for processing the growing polysaccharide for export and/or acts as a failsafe enzyme that rids the periplasm of aberrant polymer when the export process goes awry. When entire $p s l$ operon is induced by $\mathrm{P}_{\mathrm{BAD}}$ promoter and overexpressed in PAO1, Psl production is increased (Figure 1A) [6, 20]. However, Psl production is decreased when only PslG is overexpressed (Figure 1A). This suggests that the hydrolase activity or the location of PslG may be controlled by other Psl proteins in the biosynthesis apparatus of Psl to ensure that the hydrolase activity of PslG is activated only when needed. Consistently, the biofilm formation and Psl production is not affected when overexpressing the mutated PslG that has totally lost the hydrolase activity (Figure 3C and Supplementary information, Figure S4). Surprisingly, the biofilm formation is even slightly promoted by overexpression of mutated PslG (E165Q, E276Q) in PAO1 (Supplementary information, Figure S4C), which 
has mutation on two key catalytic residues. This suggests that CBM domain of PslG might be involved in the synthesis of Psl polymer.

Our previous study suggested that enzymes released from dead bacteria may degrade the polysaccharide matrix in the center of microcolony [6]. In the present study, we have revealed that such enzymes can be part of the polysaccharide synthesis apparatus. PslG is a glycosyl hydrolase predicted to be located in the periplasmic space $[23,29]$. It is an essential protein in the synthesis machinery of Psl, a key biofilm matrix exopolysaccharide in $P$. aeruginosa [23, 29]. Strikingly, overexpressed or exogenously supplied PslG prevents biofilm formation through degrading Psl. The synthesis of many polysaccharides, such as alginate and Pel, involves hydrolases [29], which could also be potential enzymes to target biofilm matrix polysaccharides. This implies a novel way for discovering anti-biofilm factors from proteins that are involved in the synthesis of matrix polysaccharides. Overall, we show that enzymes targeting biofilm matrix polysaccharides may offer a general strategy to prevent the clinical and environmental complications associated with biofilms.

\section{Materials and Methods}

\section{Strains, human cell lines, and growth conditions}

Bacterial strains and plasmids used in this study are listed in Supplementary information, Table S1. Unless otherwise indicated, P. aeruginosa was grown at $37^{\circ} \mathrm{C}$ in Luria Bertani broth (LB, Becton Dickinson) without sodium chloride (LBNS) or in Jensen's, a chemically defined medium [48]. Biofilms of P. aeruginosa were grown in Jensen's medium at $30^{\circ} \mathrm{C}$. L-arabinose (Sigma) was used as inducer for genes transcribed from $\mathrm{P}_{\mathrm{BAD}}$ promoter in $P$. aeruginosa. Biofilms of $P$. syringae and $P$. stutzeri were grown at $30{ }^{\circ} \mathrm{C}$ in $\mathrm{K}$ medium [49]. M9 medium was used to grow the biofilm of E. coli at $37{ }^{\circ} \mathrm{C}$ [50]. Biofilm of $S$. aureus was grown at $37{ }^{\circ} \mathrm{C}$ in tryptic soy broth (TSB; Becton Dickinson) with $0.25 \%$ glucose [51]; Biofilm of C. albicans was grown in Lee's medium at $25^{\circ} \mathrm{C}$ [52]; Biofilm of $S$. enteric was grown at $28^{\circ} \mathrm{C}$ in LB broth [53]. Ampicillin (Sigma) was used at $100 \mu \mathrm{g} / \mathrm{ml}$ for E. coli, while carbenicillin (Sigma) was used at $300 \mu \mathrm{g} / \mathrm{ml}$ for P. aeruginosa.

The human colonic epithelial cell line Caco2 or HT-29 cells were obtained from the American Type Culture Collection (Manassas, VA, USA). Caco2 or HT-29 cells were cultured in Dulbecco's Modified Eagle's medium (DMEM, Sigma) supplemented with $10 \%$ fetal bovine serum (FBS, Sigma), 100 units $/ \mathrm{ml}$ penicillin $\mathrm{G}$, and $100 \mu \mathrm{g} / \mathrm{ml}$ streptomycin (Sigma) at $37{ }^{\circ} \mathrm{C}$ with $5 \% \mathrm{CO}_{2}$. Tetrazolium MTT (3-[4,5-dimethylthiazol-2-yl]-2,5- diphenyltetrazolium bromide) was purchased from Sigma.

\section{Plasmid construction, protein purification and crystalliza- tion}

To construct plasmid pHERD20T- $p s l G$, the entire $p s l G$ gene was amplified from the genomic DNA of $P$. aeruginosa PAO1 and cloned into pHERD20T at EcoRI and HindIII sites [54]. For expression of PslG in E. coli, sequences encoding PslG (residues 31-442) were amplified and cloned into PGL01, a modified vector based on pET15b with a PPase cleavage site to remove the His tag. The first 30 residues of PslG were truncated because they were predicted to be a signal peptide by the Signal P4.1 server. The resulting expression plasmid PGL01-ps $l G$ was transformed into E. coli BL21 (DE3), which was cultured at $37^{\circ} \mathrm{C}$ till OD600 reached 0.8 , and induced overnight with $0.12 \mathrm{mM}$ isopropyl $\beta$-D-thiogalactopyranoside at $22{ }^{\circ} \mathrm{C}$. Substituted proteins of PslG were constructed by overlapping PCR and cloned into PGL01. The induction condition and purification procedure was the same as native protein.

For protein purification, bacterial cells were harvested by centrifugation for $15 \mathrm{~min}$ at $4200 \mathrm{rpm}$ and resuspended in binding buffer ( $25 \mathrm{mM}$ Tris- $\mathrm{HCl}, \mathrm{pH} 8.0,200 \mathrm{mM} \mathrm{NaCl})$. The bacterial suspension was lysed by sonication and centrifuged for $45 \mathrm{~min}$ at $12000 \mathrm{rpm}$ to remove the precipitate. The supernatant containing PslG was loaded onto a nickel affinity column (Chelating Sepharose Fast Flow, GE Healthcare), and washed with binding buffer for several times to remove the nonspecific proteins. The protein bound to the resin of nickel column was resuspended in binding buffer and mixed with PPase (a final concentration of $0.12 \mathrm{mg} / \mathrm{ml}$ ). The mixture was incubated overnight at $4{ }^{\circ} \mathrm{C}$ to remove the His tag. The protein sample was then eluted with binding buffer. For further purification, PslG was loaded onto an ion-exchange column (Source 15Q HR 16/10, GE Healthcare) and eluted with a linear gradient of $0-1 \mathrm{M} \mathrm{NaCl}$ in $25 \mathrm{mM}$ Tris- $\mathrm{HCl}$ buffer $\mathrm{pH}$ 8.0. Finally, the protein sample was purified by size-exclusion chromatography (Superdex 20010/300 GL, GE Healthcare) with $10 \mathrm{mM}$ Tris-HCl buffer $\mathrm{pH} 8.0$ containing $100 \mathrm{mM} \mathrm{NaCl}$. The protein was then further purified by ion exchange (Source 15QHR 16/10, GE Healthcare) and size-exclusion chromatography (Superdex 200 10/300 GL, GE Healthcare). Selenomethionine (Se-Met)-labeled PslG was obtained by culturing BL21(DE3)/PGL01-pslG in M9 medium supplemented with selenomethionnine. The induction condition and purification procedure was the same as native protein.

Preliminary screenings were performed with Hampton Research crystal screen kits by sitting drop vapor diffusion at $20{ }^{\circ} \mathrm{C}$. A total of 720 conditions from 15 kits were tested. Briefly, equal volumes of protein and reservoir solution were mixed together. Initial crystals were optimized using hanging drop method to increase the diffraction. The best native crystals were obtained under conditions of 3\% (w/v) Dextran sulfate sodium salt, $0.1 \mathrm{M}$ Bicine $\mathrm{pH} 8.5$ and $7 \%(\mathrm{w} / \mathrm{v})$ PEG20000. The Se-Met-labeled crystals were obtained under conditions of $2 \%(\mathrm{v} / \mathrm{v})$ Tacsimate $\mathrm{pH}$ 8.0, 0.1 M Tris$\mathrm{HCl} \mathrm{pH} 8.5$ and $15 \%$ w/v PEG3350. Crystals with good diffraction quality were soaked in a solvent identical to the reservoir solution with $20 \%(\mathrm{v} / \mathrm{v})$ glycerol, and flash frozen in liquid nitrogen. All data were collected on beamline BL17U1 at Shanghai Synchrotron Radiation Facility (SSRF).

\section{Biofilm inhibition or disassembly assay}

PslG was supplied into the growth media at inoculation in the biofilm inhibition assay. For biofilm disassembly, the culture was replaced by fresh media containing PslG after 36-h growth for flow-cell biofilms, $30 \mathrm{~h}$ for biofilms grown in microfluidic devices or 24-h growth in glass chamber (pellicles) or in microtiter dishes. Unless otherwise indicated, PslG was supplied at $50 \mathrm{nM}$ final concentration. For PslG treatment performed in microtiter dishes (Falcon 3911), a 1/100 dilution of an overnight culture was used for 
inoculation. For biofilm inhibition assay, the medium and planktonic cells were discarded after $24 \mathrm{~h}$ of static growth at $30^{\circ} \mathrm{C}$, and the wells were washed and then stained with $0.1 \%$ crystal violet (CV, Sigma) solution. For biofilm disassembly, the biofilms were washed and stained by $\mathrm{CV}$ after $30 \mathrm{~min}$ or hours of PslG treatment at $30{ }^{\circ} \mathrm{C}$ or the indicated temperatures. The $\mathrm{CV}$ bound to biofilms was solubilized with $30 \%$ acetic acid and measured for absorbance at $560 \mathrm{~nm}$. The sample without PslG was used as an untreated control. The inhibition rate or disassembly rate were calculated using the formula $=\left[\left(\mathrm{A}_{560}\right.\right.$ of untreated control $-\mathrm{A}_{560}$ of PslG-treated sample) $/ \mathrm{A}_{560}$ of untreated control] $\times 100 \%$. Two tailed $t$-test was performed to discern differences between groups.

Image acquisition and analysis of flow-cell biofilms and air-liquid interface biofilms (pellicles)

The air-liquid interface biofilms, pellicles were grown in glass chambers with individual chamber dimensions of $1 \times 1 \times 4 \mathrm{~cm}$ (Chambered \#1.5 German Coverglass System, Nunc Inc.) as described previously [7]. For confocal laser scanning microscopy (CLSM) observation, buffer was gently removed from glass chambers to allow the pellicles to drop onto coverslips. The flowcell biofilms were grown at RT in three-channel flow cell with individual channel dimensions of $1 \times 4 \times 40 \mathrm{~mm}$ (Stovall Life Science, Inc.) as previously described [20]. The mid-log phase culture was used for inoculation. The biofilms were stained with DNA stain SYTO9 (Molecular Probes, Invitrogen). The Psl matrix was stained with fluorescence-labeled lectin HHA at $100 \mu \mathrm{g} / \mathrm{ml}$ (EY lab, INC) as we described elsewhere [6]. The fluorescent and differential interference contrast (DIC) images were acquired with a FV1000 confocal laser scanning microscope (Olympus, Japan) or a Leica TCS SP8 (Leica Microsystems, Japan). The biofilm biomass and thickness were quantified by COMSTAT software [55].

\section{Psl extract preparation and degradation of Psl in vitro by PslG}

Psl polysaccharide extract was prepared from overnight cultures as previously described [23] with modifications. To remove the DNA, crude Psl polysaccharide was precipitated with three volumes of ethanol and dissolved in pyrogen-free distilled water with $5 \mathrm{mM} \mathrm{MgCl}$, and subsequently treated by $0.1 \mathrm{mg} / \mathrm{ml}$ DNase $\mathrm{I}$ (Sigma) for $5 \mathrm{~h}$ at $37^{\circ} \mathrm{C}$ and $0.1 \mathrm{mg} / \mathrm{ml}$ proteinase $\mathrm{K}$ for $1 \mathrm{~h}$ at 60 ${ }^{\circ} \mathrm{C}$. Enzymes were inactivated at $80{ }^{\circ} \mathrm{C}$ for $30 \mathrm{~min}$. To test the Psl degradation by PslG, Psl ( $4 \mathrm{mg} / \mathrm{ml}$ and lower) were incubated with $50 \mathrm{nM}$ PslG for $1 \mathrm{~h}$ at different temperatures. Treated or untreated samples were examined by immunoblotting against anti-Psl serum as previously described [23]. Densitometry software Quantity One (Bio-Rad) was used to quantify the immunoblotting data. Psl concentration of each dot was calculated by the corresponding standard curves. The catalytic productivity of PslG was defined as the amount of Psl degraded per nmol PslG per hour indicated by the formula: [(Psl concentration before treatment - Psl concentration after treatment $) /($ Treating time $\times$ PslG concentration $)]$.

\section{Biofilm dispersal assay in the microfluidic devices}

The microfluidic devices were fabricated using soft lithography technique [56]. The top PDMS layer contained 10 microchannels (8 mm long, $60 \mu \mathrm{m}$ in height, and $300 \mu \mathrm{m}$ in width) as illustrated in Figure 5C. Holes were punched to make inlets and outlets of the channels using a sharpened 21-gauge needle. After oxygen plasma treatment (Harrick Plasma, Ithaca, NY, USA), and being baked at
$110{ }^{\circ} \mathrm{C}$ for $1 \mathrm{~h}$, the top layer was permanently bonded with a bottom glass slide ( $1 \mathrm{~mm}$ in thickness). Prior to inoculation, the device was disinfected with $75 \%$ ethanol for $15 \mathrm{~min}$ and then rinsed with sterile distilled water for $15 \mathrm{~min}$. Then $1 \mathrm{ml}$ plastic syringes (ZYMM, Shanghai, China) with needle attached to 30-gauge Teflon tubing (Zeus Inc., Orangeburg, SC) were connected with the microchannels via inlets, for feeding samples. Bacterial suspension and testing media were fed into the microchannels at a flow rate of $0.3 \mu \mathrm{l} / \mathrm{min}$, unless declared otherwise.

GFP-tagged PAO1 culture (OD600 $=0.4)$ was used to inoculate microchannels and incubated for $2 \mathrm{~h}$ at RT to allow bacteria adhere to the glass surface. Following incubation, Jensen media was injected along the same channel as the inoculum. Biofilms were formed after $\sim 30 \mathrm{~h}$. Then the media containing PslG, antibiotics, or combination, were introduced through the inlets for $5 \mathrm{~h}$. The working concentration of tobramycin and ciprofloxacin is $0.1 \mathrm{mg} / \mathrm{ml}$. The biomass values of bacterial biofilms were determined by fluorescence imaging using an Eclipse Ti inversed microscope (Nikon, Japan), equipped with a NEO sCMOS CCD camera (Andor, UK). The biomass values of microfluidic biofilms were determined by Image J software.

\section{MIC and MBEC measurements}

MICs of planktonic and PslG-dispersed bacteria were determined by E test strips (AB Biodisk) following the manufacturer's instruction. The measurement of MBEC and MIC of Peg-biofilms were performed on Calgary Biofilm Device (CBD) following the instruction of the $\mathrm{MBEC}^{\mathrm{TM}}$ high-throughput assay (Innovotech) with modifications. Briefly, identical biofilms were formed on plastic pegs on the lid of the CBD. Peg-biofilms were exposed to media or media containing $50 \mathrm{nM}$ PslG for $1 \mathrm{~h}$ before challenge of serial antibiotics. The MIC represents the concentration of antibiotic required to inhibit growth of a planktonic bacterial population from Peg-biofilms (OD600 $<0.1)$ and the MBEC value is the lowest dilution that prevents re-growth of bacteria from the treated biofilms $(\mathrm{OD} 600<0.1)$.

\section{Mouse implant infection model}

Experiments using animal models were performed in accordance with the ARF-SBS/NIE NTU Institutional Animal Care and Use Committee (IACUC) ethics approval and guidelines (protocol no.ARF SBS/NIE-A0191). PAO1 biofilms were initially established on cylindrical silicone implants $(3 \mathrm{~mm} \times 5 \mathrm{~mm})$ in $0.9 \% \mathrm{NaCl}$ at $37^{\circ} \mathrm{C}$ with shaking at $110 \mathrm{rpm}$ for $20 \mathrm{~h}$. 7-week-old female BALC/C mice were used and anesthetized with $100 \mathrm{mg} / \mathrm{kg}$ ketamine and $10 \mathrm{mg} / \mathrm{kg}$ xylene. The biofilm-coated implants were washed with $0.9 \% \mathrm{NaCl}$ and transferred into the peritoneum of the mice. PslG and tobramycin were injected at the site of implantation after $2 \mathrm{~h}$. Four groups of five mice were treated with $0.9 \%$ $\mathrm{NaCl}$ (as control), $50 \mathrm{nM}$ PslG, $50 \mathrm{mg} / \mathrm{kg}$ tobramycin, and $50 \mathrm{nM}$ $\mathrm{PslG}+50 \mathrm{mg} / \mathrm{kg}$ tobramycin, respectively. After $24 \mathrm{~h}$ of infection, the mice were sacrificed to harvest the implants. The implants were sonicated in $1 \mathrm{ml} 0.9 \% \mathrm{NaCl}$ in an ice water bath for $10 \mathrm{~min}$ to disrupt and homogenize the biofilm cells from the implants. The samples were then diluted serially and grown on LB agar at $37^{\circ} \mathrm{C}$ for $20 \mathrm{~h}$. CFU $/ \mathrm{ml}=$ average number of colonies $\times$ dilution factor $\times$ volume used to plate on LB agar.

\section{Structure determination and refinement}

Both native and anomalous data sets were processed using 
HKL2000 software suite [57]. The structure of PslG was solved by single-wavelength anomalous dispersion (SAD) phasing. The initial model was automatically built using PHENIX [58]. One monomer was then used as the search model in the determination of the native structure by molecular replacement using PHASE $[59,60]$. The native structure was further refined interactively by using PHENIX and COOT [61]. All diffraction data and structure refinement statistics were summarized in Table 1. Structural representations were generated using online software PyMOL (http:// www.pymol.org).

\section{Molecular docking simulation and modeling}

The program AutoDock 4.2 [62] was used for the molecular docking calculations. $\beta$-D-Glcp- $(1 \rightarrow 3)-[\alpha-\mathrm{D}-\mathrm{Manp}-(1 \rightarrow 2)]-\beta-\mathrm{D}$ Manp-(1 $\rightarrow 3)-\beta-\mathrm{D}-$ Manp-(1-1)-OH, the partial structure model of Psl polysaccharide repeat unit, was generated by the Glycam Biomolecule Builder on GLYCAM Web (http://www.glycam.com). The ligand and receptor were prepared by AutoDock Tools. All rotatable bonds of the ligand were accepted and the receptor was assumed to be rigid. Polar hydrogens and Kollman united atom charges were added to the enzyme, while gasteiger charges were assigned and nonpolar hydrogen atoms were merged for the ligand. The docking calculations were carried out by using the default settings for the Genetic algorithm parameters with 25000000 energy evaluations per run. A composite file of all possible conformers was analyzed by AutoDock Tools. The chemical reasonableness of the best results was evaluated by examining the interactions between the receptor and the best-docked conformers.

\section{Macrophage cytotoxicity assay}

As previously described [63], $5 \times 10^{5}$ RAW264.7 macrophages were grown in 24-well plates (Nunc, Denmark) and incubated at $37{ }^{\circ} \mathrm{C}, 5 \% \mathrm{CO}_{2}$ for $16 \mathrm{~h}$. The macrophages were then washed twice with phosphate-buffered saline (PBS) and incubated with 0 , 50, 100, 200, and $500 \mathrm{nM}$ PslG in DMEM at $37{ }^{\circ} \mathrm{C}, 5 \% \mathrm{CO}_{2}$ for $24 \mathrm{~h}$. The toxicity of the PslG was determined by monitoring cell viability with $20 \mu \mathrm{M}$ propidium iodide. Cells that were observed unstained or stained red under the inverted epifluorescent microscope (Zeiss) were counted as live or dead, respectively. Cells from 5 images were enumerated for the ratio of dead cells to live cells calculation. Experiments were performed in triplicates, and the results are shown as the mean $\pm \mathrm{SD}$.

\section{Macrophage-biofilm interaction assay}

The assay was done as previously described with modifications [63]. Briefly, P. aeruginosa PAO1 biofilm was grown on sterile glass coverslips in minimal medium at $37{ }^{\circ} \mathrm{C}$ for $24 \mathrm{~h}$. The biofilms were washed twice in $0.9 \% \mathrm{NaCl}$ to remove unattached cells and aggregates and then treated with 0 or $50 \mathrm{nM}$ PslG in minimal medium at $37{ }^{\circ} \mathrm{C}$ for $1 \mathrm{~h}$. The treated biofilms were then washed twice in $0.9 \% \mathrm{NaCl}$ and further incubated with $5 \times 10^{5}$ RAW264.7 macrophages in DMEM at $37{ }^{\circ} \mathrm{C}, 5 \% \mathrm{CO}_{2}$ for $2 \mathrm{~h}$. After washing away the dispersed bacterial cells in $0.9 \% \mathrm{NaCl}$, the remaining biofilms were scraped off the glass slides and re-suspended in $1 \mathrm{ml}$ $0.9 \% \mathrm{NaCl}$. Serial dilutions of the bacterial cell suspension were conducted, and the diluted bacterial suspensions were plated on $\mathrm{LB}$ agar. After incubation at $37^{\circ} \mathrm{C}$ for $18 \mathrm{~h}, \mathrm{CFU}$ were enumerated and tabulated. Experiments were performed in triplicates, and the results are shown as the mean $\pm \mathrm{SD}$.
MTT cell cytotoxicity assay

Cell cytotoxicity of PslG was performed by MTT assay as previously described [64]. Briefly, Caco-2 or HT-29 cells were seeded to a 96 -well plate at $10^{4}$ cells/well and incubated for $12 \mathrm{~h}$, followed by incubation with fresh DMEM media containing various concentrations of PslG for $12 \mathrm{~h}$. Then, $10 \mu \mathrm{l}$ of $5 \mathrm{mg} / \mathrm{ml}$ MTT was added to each well and the plate was incubated for another $4 \mathrm{~h}$ at $37{ }^{\circ} \mathrm{C}$ to allow MTT metabolism into water insoluble purple formazan crystals. The formazan crystals were finally solubilized by adding $100 \mu \mathrm{l}$ of DMSO. The optical density at $540 \mathrm{~nm}$ was measured using Synergy H1 Hybrid microplate Reader (BioTek Instruments, Inc.). The assays were conducted three times independently.

\section{C. elegans liquid killing assay}

To compare the virulence between planktonic bacteria and PslG-dispersed biofilm bacteria, we conducted a liquid killing assay using $C$. elegans as the model host. The assay was performed as previously described with modifications [63]. The PslG-dispersed bacterial cells were collected from PAO1 pellicles grown in Jensen's medium for $24 \mathrm{~h}$. PslG was used at $50 \mathrm{nM}$. Planktonic and dispersed bacteria were inoculated in liquid assay media in 96-well microtiter plates. Thirty of L4-stage $C$. elegans were transferred into each well and co-cultured for $48 \mathrm{~h}$ at $25^{\circ} \mathrm{C}$. Then live/dead nematodes were observed and determined under a stereomicroscope (Olympus). Experiments were performed in triplicate, and the results are shown as the mean $\pm \mathrm{SD}$.

\section{Dynamic light scattering}

The DLS measurement of PslG was performed using a DynaPro-NanoStar SN271-DPN instrument with temperature control at $25{ }^{\circ} \mathrm{C}$ (Protein Solutions). The protein sample was prepared in 10 $\mathrm{mM}$ Tris- $\mathrm{HCl} \mathrm{pH} 8.0,100 \mathrm{mM} \mathrm{NaCl}$. The concentration of PslG used for DLS measurements was $2.5 \mathrm{mg} / \mathrm{ml}$. The DLS result was analyzed using the software DYNAMICS v.7.1.5.

\section{Detecting the effect of PslG on the biofilm of PAOl/pC- $d r A: \because g f p^{s}$}

The pellicles of PAO1/pCdrA::gfp were grown in Jensen's medium in the wells of a Costar 96-well cluster plate (Corning Life Sciences), and then the culture was replaced by fresh media or media containing $50 \mathrm{nM}$ PslG. The green fluorescent signal was measured and recorded every $10 \mathrm{~min}$ for the following $3 \mathrm{~h}$ using $\lambda \mathrm{ex} / \lambda \mathrm{cm}$ of $488 / 520 \mathrm{~nm}$ by Synergy H4 Hydrid Reader (BioTek).

\section{Accession code}

Coordinate and structure factor files have been deposited in the PDB (http://www.rcsb.org/pdb) under ID code 4ZN2.

\section{Acknowledgments}

We thank DJ Wozniak and MR Parsek for providing strains, antibodies and plasmids; L Howell and P Baker for sharing data; C Peng and F Li for carbohydrate analysis. This work was supported by the National Basic Research Program of China (973 Program; 2014CB846002 to LM, and 2015CB150600 to LG and LM), and the National Natural Science Foundation of China (31270177 to LM, 31470732 to LG, and 31200044 to DW). We have filed a patent application on the use of PslG. 


\section{References}

1 Costerton JW, Lewandowski Z, Caldwell DE, Korber DR, Lappin-Scott HM. Microbial biofilms. Annu Rev Microbiol 1995; 49:711-745.

2 Stoodley P, Sauer K, Davies DG, Costerton JW. Biofilms as complex differentiated communities. Annu Rev Microbiol 2002; 56:187-209.

3 Stewart PS, Franklin MJ. Physiological heterogeneity in biofilms. Nat Rev Micro 2008; 6:199-210.

4 Stewart PS, Costerton JW. Antibiotic resistance of bacteria in biofilms. The Lancet 2001; 358:135-138.

5 Lewis K. Persister cells, dormancy and infectious disease. Nat Rev Micro 2007; 5:48-56.

6 Ma L, Conover M, Lu H, Parsek MR, Bayles K, Wozniak DJ. Assembly and development of the Pseudomonas aeruginosa biofilm matrix. PLoS Pathog 2009; 5:e1000354.

7 Wang S, Parsek MR, Wozniak DJ, Ma LZ. A spider web strategy of type IV pili-mediated migration to build a fibre-like Psl polysaccharide matrix in Pseudomonas aeruginosa biofilms. Environ Microbiol 2013; 15:2238-2253.

8 Billings N, Millan M, Caldara M, et al. The extracellular matrix component Psl provides fast-acting antibiotic defense in Pseudomonas aeruginosa biofilms. PLoS Pathog 2013; 9:e1003526.

9 Mishra M, Byrd MS, Sergeant S, et al. Pseudomonas aeruginosa Psl polysaccharide reduces neutrophil phagocytosis and the oxidative response by limiting complement-mediated opsonization. Cell Microbiol 2012; 14:95-106.

10 Newell PD, Boyd CD, Sondermann H, O'Toole GA. A c-diGMP effector system controls cell adhesion by inside-out signaling and surface protein cleavage. PLoS Biol 2011; 9:e1000587.

11 Kiedrowski MR, Kavanaugh JS, Malone CL, et al. Nuclease modulates biofilm formation in community-associated methicillin-resistant Staphylococcus aureus. PLoS One 2011; 6:e26714.

12 Moormeier DE, Bose JL, Horswill AR, Bayles KW. Temporal and stochastic control of Staphylococcus aureus biofilm development. mBio 2014; 5:e1341.

13 Govan JRW, Deretic V. Microbial pathogenesis in cystic fibrosis: mucoid Pseudomonas aeruginosa and Burkholderia cepacia. Microbiol Rev 1996; 60:539-574.

14 Lyczak JB, Cannon C, Pier GB. Establishment of Pseudomonas aeruginosa infection: lessons from a versatile opportunist. Microbes Infect 2000; 2:1051-1060.

15 Singh PK, Schaefer AL, Parsek MR, Moninger TO, Welsh MJ, Greenberg EP. Quorum-sensing signals indicate that cystic fibrosis lungs are infected with bacterial biofilms. Nature 2000; 407:762-764.

16 Fazli M, Bjarnsholt T, Kirketerp-Møller K, et al. Nonrandom distribution of Pseudomonas aeruginosa and Staphylococcus aureus in chronic wounds. J Clin Microbiol 2009; 47:40844089.

17 Colvin KM, Irie Y, Tart CS, et al. The Pel and Psl polysaccharides provide Pseudomonas aeruginosa structural redundancy within the biofilm matrix. Environ Microbiol 2012; 14:19131928.

18 Branda SS, Vik A, Friedman L, Kolter R. Biofilms: the matrix revisited. Trends Microbiol 2005; 13:20-26.

19 Ramsey DM, Wozniak DJ. Understanding the control of Pseudomonas aeruginosa alginate synthesis and the prospects for management of chronic infections in cystic fibrosis. Mol Microbiol 2005; 56:309-322.

20 Ma L, Jackson KD, Landry RM, Parsek MR, Wozniak DJ. Analysis of Pseudomonas aeruginosa conditional Psl variants reveals roles for the Psl polysaccharide in adhesion and maintaining biofilm structure postattachment. J Bacteriol 2006; 188:8213-8221.

21 Wozniak DJ, Wyckoff TJO, Starkey M, et al. Alginate is not a significant component of the extracellular polysaccharide matrix of PA14 and PAO1 Pseudomonas aeruginosa biofilms. Proc Natl Acad Sci USA 2003; 100:7907-7912.

22 Friedman L, Kolter R. Genes involved in matrix formation in Pseudomonas aeruginosa PA14 biofilms. Mol Microbiol 2004; 51:675-690.

23 Byrd MS, Sadovskaya I, Vinogradov E, et al. Genetic and biochemical analyses of the Pseudomonas aeruginosa Psl exopolysaccharide reveal overlapping roles for polysaccharide synthesis enzymes in Psl and LPS production. Mol Microbiol 2009; 73:622-638.

24 Yang L, Hu Y, Liu Y, Zhang J, Ulstrup J, Molin S. Distinct roles of extracellular polymeric substances in Pseudomonas aeruginosa biofilm development. Environ Microbiol 2011; 3:1705-1717.

25 Yang L, Hengzhuang W, Wu H, et al. Polysaccharides serve as scaffold of biofilms formed by mucoid Pseudomonas aeruginosa. FEMS Immunol Med Microbiol 2012; 65:366-376.

26 Tseng BS, Zhang W, Harrison JJ, et al. The extracellular matrix protects Pseudomonas aeruginosa biofilms by limiting the penetration of tobramycin. Environ Microbiol 2013; 15:2865-2878.

27 Ma L, Wang S, Wang D, Parsek MR, Wozniak DJ. The roles of biofilm matrix polysaccharide Psl in mucoid Pseudomonas aeruginosa biofilms. FEMS Immunol Med Microbiol 2012; 65:377-380.

28 Irie Y, Borlee BR, O'Connor JR, et al. Self-produced exopolysaccharide is a signal that stimulates biofilm formation in Pseudomonas aeruginosa. Proc Natl Acad Sci USA 2012; 109:20632-20636.

29 Franklin MJ, Nivens DE, Weadge JT, Howell PL. Biosynthesis of the Pseudomonas aeruginosa extracellular polysaccharides, alginate, Pel, and Psl. Front Microbiol 2011; 2:167.

30 Rybtke MT, Borlee BR, Murakami K, et al. Fluorescence-based reporter for gauging cyclic di-GMP levels in Pseudomonas aeruginosa. Appl Environ Microbiol 2012; 78:5060-5069.

31 Ma L, Wang J, Wang S, et al. Synthesis of multiple Pseudomonas aeruginosa biofilm matrix exopolysaccharides is post-transcriptionally regulated. Environ Microbiol 2012; 14:1995-2005.

32 Whitchurch CB, Tolker-Nielsen T, Ragas PC, Mattick JS. Extracellular DNA required for bacterial biofilm formation. Science 2002; 295:1487.

33 Holm L, Rosenstrom P. Dali server: conservation mapping in 3D. Nucleic Acids Res 2010; 38:W545-549.

34 Larson SB, Day J, Barba de la Rosa AP, Keen NT, McPherson A. First crystallographic structure of a xylanase from glyco- 
side hydrolase family 5: implications for catalysis. Biochemistry 2003; 42:8411-8422.

35 Kobata A. Exo- and endoglycosidases revisited. Proc Jpn Acad Ser B Phys Biol Sci 2013; 89:97-117.

36 Koshland DE. Stereochemistry and the mechanism of enzymatic reactions. Biol Rev 1953; 28:416-436.

37 Davies G, Henrissat B. Structures and mechanisms of glycosyl hydrolases. Structure 1995; 3:853-859.

38 Starkey M, Hickman JH, Ma L, et al. Pseudomonas aeruginosa rugose small-colony variants have adaptations that likely promote persistence in the cystic fibrosis lung. J Bacteriol 2009; 191:3492-3503.

39 Ceri H, Olson ME, Stremick C, Read RR, Morck D, Buret A. The Calgary Biofilm Device: new technology for rapid determination of antibiotic susceptibilities of bacterial biofilms. $J$ Clin Microbiol 1999; 37:1771-1776.

40 Kolodkin-Gal I, Romero D, Cao S, Clardy J, Kolter R, Losick R. D-amino acids trigger biofilm disassembly. Science 2010; 328:627-629.

41 Xiao J, Klein MI, Falsetta ML, et al. The exopolysaccharide matrix modulates the interaction between 3D architecture and virulence of a mixed-species oral biofilm. PLoS Pathog 2012; 8:e1002623.

42 Mitchell KF, Zarnowski R, Sanchez H, et al. Community participation in biofilm matrix assembly and function. Proc Natl Acad Sci USA 2015; 112:4092-4097.

43 Kaplan JB, Meyenhofer MF, Fine DH. Biofilm growth and detachment of Actinobacillus actinomycetemcomitans. J Bacteriol 2003; 185:1399-1404.

44 Kaplan JB, Ragunath C, Ramasubbu N, Fine DH. Detachment of Actinobacillus actinomycetemcomitans biofilm cells by an endogenous beta-hexosaminidase activity. J Bacteriol 2003; 185:4693-4698.

45 Leiman SA, May JM, Lebar MD, Kahne D, Kolter R, Losick R. D-amino acids indirectly inhibit biofilm formation in Bacillus subtilis by interfering with protein synthesis. J Bacteriol 2013; 195:5391-5395.

46 Nguyen D, Joshi-Datar A, Lepine F, et al. Active starvation responses mediate antibiotic tolerance in biofilms and nutrient-limited bacteria. Science 2011; 334:982-987.

47 Roske Y, Sunna A, Pfeil W, Heinemann U. High-resolution crystal structures of Caldicellulosiruptor strain Rt8B.4 carbohydrate-binding module CBM27-1 and its complex with mannohexaose. J Mol Biol 2004; 340:543-554.

48 Jensen SE, Facycz IT, Campbell JN. Nutritional factors controlling exocellular protease production by Pseudomonas aeruginosa. J Bacteriol 1980; 144:844-847.

49 Franche C, Elmerich C. Physiological properties and plasmid content of several strains of Azospirillum brasilense and $A$. lipoferum. Ann Microbiol (Paris) 1981; 132A:3-18.

50 Schembri MA, Klemm P. Biofilm formation in a hydrodynamic environment by novel fimh variants and ramifications for virulence. Infect Immun 2001; 69:1322-1328.

51 Christensen GD, Simpson WA, Younger JJ, et al. Adherence of coagulase-negative staphylococci to plastic tissue culture plates: a quantitative model for the adherence of staphylococci to medical devices. J Clin Microbiol 1985; 22:996-1006.
52 Sahni N, Yi S, Daniels KJ, Huang G, Srikantha T, Soll DR. Tec1 mediates the pheromone response of the white phenotype of Candida albicans: insights into the evolution of new signal transduction pathways. PLoS Biol 2010; 8:e1000363.

53 Aya Castaneda Mdel R, Sarnacki SH, Noto Llana M, Lopez Guerra AG, Giacomodonato MN, Cerquetti MC. Dam methylation is required for efficient biofilm production in Salmonella enterica serovar Enteritidis. Int J Food Microbiol 2015; 193:15-22.

54 Qiu D, Damron FH, Mima T, Schweizer HP, Yu HD. PBADbased shuttle vectors for functional analysis of toxic and highly regulated genes in Pseudomonas and Burkholderia spp. and other bacteria. Appl Environ Microbiol 2008; 74:7422-7426.

55 Heydorn A, Nielsen AT, Hentzer M, et al. Quantification of biofilm structures by the novel computer program COMSTAT. Microbiol 2000; 146 (Pt10):2395-2407.

56 Duffy DC, McDonald JC, Schueller OJ, Whitesides GM. Rapid prototyping of microfluidic systems in poly(dimethylsiloxane). Anal Chem 1998; 70:4974-4984.

57 Otwinowski Z, Minor W. [20] Processing of X-ray diffraction data collected in oscillation mode. Methods Enzymol 1997; 276:307-326

58 Adams PD, Grosse-Kunstleve RW, Hung LW, et al. PHENIX: building new software for automated crystallographic structure determination. Acta Crystallogr D Biol Crystallogr 2002; 58:1948-1954.

59 McCoy AJ, Grosse-Kunstleve RW, Adams PD, Winn MD, Storoni LC, Read RJ. Phaser crystallographic software. J Appl Crystallogr 2007; 40:658-674.

60 Winn MD, Ballard CC, Cowtan KD, et al. Overview of the CCP4 suite and current developments. Acta Crystallogr D Biol Crystallogr 2011; 67:235-242.

61 Emsley P, Cowtan K. Coot: model-building tools for molecular graphics. Acta Crystallogr D Biol Crystallogr 2004; 60:2126-2132.

62 Morris GM, Huey R, Lindstrom W, et al. AutoDock4 and AutoDockTools4: Automated docking with selective receptor flexibility. J Comput Chem 2009; 30:2785-2791.

63 Chua SL, Liu Y, Yam JK, et al. Dispersed cells represent a distinct stage in the transition from bacterial biofilm to planktonic lifestyles. Nat Commun 2014; 5:4462.

64 Mosmann T. Rapid colorimetric assay for cellular growth and survival: application to proliferation and cytotoxicity assays. J Immunol Methods 1983; 65:55-63.

65 Rahme LG, Stevens EJ, Wolfort SF, Shao J, Tompkins RG, Ausubel FM. Common virulence factors for bacterial pathogenicity in plants and animals. Science 1995; 268:1899-1902.

66 Holloway BW. Genetic recombination in Pseudomonas aeruginosa. J Gen Microbiol 1955; 13:572-581.

67 Wolfgang MC, Kulasekara BR, Liang X, et al. Conservation of genome content and virulence determinants among clinical and environmental isolates of Pseudomonas aeruginosa. Proc Natl Acad Sci USA 2003; 100:8484-8489.

68 Mathee K, Ciofu O, Sternberg C, et al. Mucoid conversion of Pseudomonas aeruginosa by hydrogen peroxide: a mechanism for virulence activation in the cystic fibrosis lung. Microbiol 1999; 145 (Pt 6):1349-1357.

(Supplementary information is linked to the online version of the paper on the Cell Research website.) 